\title{
General Raina fractional integral inequalities on coordinates of convex functions
}

\author{
Dumitru Baleanu ${ }^{1,2,3}$, Artion Kashuri ${ }^{4}$, Pshtiwan Othman Mohammed ${ }^{5 *}$ (D) and Badreddine Meftah ${ }^{6}$
}

"Correspondence:

pshtiwansangawi@gmail.com

${ }^{5}$ Department of Mathematics, College of Education, University of

Sulaimani, Sulaimani, Kurdistan Region, Iraq

Full list of author information is available at the end of the article

\begin{abstract}
Integral inequality is an interesting mathematical model due to its wide and significant applications in mathematical analysis and fractional calculus. In this study, authors have established some generalized Raina fractional integral inequalities using an $\left(l_{1}, h_{1}\right)-\left(l_{2}, h_{2}\right)$-convex function on coordinates. Also, we obtain an integral identity for partial differentiable functions. As an effect of this result, two interesting integral inequalities for the $\left(I_{1}, h_{1}\right)-\left(I_{2}, h_{2}\right)$-convex function on coordinates are given. Finally, we can say that our findings recapture some recent results as special cases.
\end{abstract}

MSC: 26D07; 26D10;26D15

Keywords: Hermite-Hadamard inequality; Raina fractional integral operators; Coordinated convex function

\section{Introduction}

In the past two decades, fractional calculus has received much attention. The fast interest in the topic is due to its extensive applications in various fields such as biochemistry, physics, viscoelasticity, fluid mechanics, computer modeling, and engineering, see [1-3] for further detail. Most of the studies have been devoted to the existence and uniqueness of solutions for fractional differential equations (FDEs); see e.g. [4-9]. A fractional differential equation needs a certain inequality to be existent and unique for solution. For this reason, a huge number of mathematicians have competed to seek such inequalities; see e.g. [10-29].

Always, it is important and necessary to specify which model or definition is being used because there are many different ways of defining fractional integrals and derivatives. To further facilitate the discussion of this model, we present here the definition which is most commonly used for fractional integrals and derivatives, namely the Riemann-Liouville (RL) definition.

Definition 1.1 $([1,2])$ For any $\mathrm{L}^{1}$ function $f(x)$ on an interval $\left[\chi_{1}, \chi_{2}\right]$ with $x \in\left[\chi_{1}, \chi_{2}\right]$, the $\eta$ th left-RL fractional integral of $f(x)$ is defined as follows:

$$
{ }^{\mathrm{RL}} \mathrm{J}_{\chi_{1}+}^{\eta} f(x):=\frac{1}{\Gamma(\eta)} \int_{\chi_{1}}^{x}(x-\xi)^{\eta-1} f(\xi) d \xi, \quad \chi_{1}<x,
$$

(c) The Author(s) 2021. This article is licensed under a Creative Commons Attribution 4.0 International License, which permits use, sharing, adaptation, distribution and reproduction in any medium or format, as long as you give appropriate credit to the original author(s) and the source, provide a link to the Creative Commons licence, and indicate if changes were made. The images or other third party material in this article are included in the article's Creative Commons licence, unless indicated otherwise in a credit line to the material. If material is not included in the article's Creative Commons licence and your intended use is not permitted by statutory regulation or exceeds the permitted use, you will need to obtain permission directly from the copyright holder. To view a copy of this licence, visit http://creativecommons.org/licenses/by/4.0/. 
for $\operatorname{Re}(\eta)>0$. Also, the $\eta$ th right-RL fractional integral of $f(x)$ is defined as follows:

$$
\mathrm{RL}_{\mathcal{J}_{2}}^{\eta} f(x):=\frac{1}{\Gamma(\eta)} \int_{x}^{\chi_{2}}(\xi-x)^{\eta-1} f(\xi) d \xi, \quad x<\chi_{2} .
$$

In the recent decades, a strong modern direction of research in fractional calculus has brought the attention of interested researchers in various disciplines to investigate various possible ways to define fractional integrals and derivatives, often with different properties from the classical RL in Definition 1.1. In 2005, Raina [30] introduced the new fractional integrals, often called the Raina fractional integrals, corresponding to the classical RL integrals (1.1) and (1.2).

Definition 1.2 ([30]) For any $\mathrm{L}^{1}$ function $f(x)$ on an interval $\left[\chi_{1}, \chi_{2}\right]$ with $x \in\left[\chi_{1}, \chi_{2}\right]$, the $\eta$ th left Raina fractional integral of $f(x)$ is defined as follows:

$$
\Im_{\rho, \eta, \chi_{1}^{+}, \omega}^{\sigma} \varphi(x)=\int_{\chi_{1}}^{x}(x-t)^{\eta-1} \mathfrak{F}_{\rho, \eta}^{\sigma}\left[\omega(x-t)^{\rho}\right] \varphi(t) d t, \quad \chi_{1}<x,
$$

and the $\eta$ th right Raina fractional integral of $f(x)$ is defined as follows:

$$
\mathfrak{\Im}_{\rho, \eta, \chi_{2}^{-}, \omega}^{\sigma} \varphi(x)=\int_{x}^{\chi_{2}}(t-x)^{\eta-1} \mathfrak{F}_{\rho, \eta}^{\sigma}\left[\omega(t-x)^{\rho}\right] \varphi(t) d t, \quad x<\chi_{2},
$$

where $\mathfrak{F}_{\rho, \eta}^{\sigma}(x)$ is the generalization of Mittag-Leffler (ML) function defined as follows: For a bounded arbitrary sequence $\sigma(k)$ of real or complex numbers, we define the function $\mathfrak{F}_{\rho, \eta}^{\sigma}(x)$ by

$$
\mathfrak{F}_{\rho, \eta}^{\sigma}(x)=\sum_{k=0}^{\infty} \frac{\sigma(k)}{\Gamma(\rho k+\eta)} x^{k}
$$

where $\rho, \eta \in \mathbb{C}$ with $\operatorname{Re}(\rho)>0, x \in \mathbb{R}$, and $\Gamma(\cdot)$ denotes the classical gamma function.

Remark 1.1 By making use of $\eta=\alpha, \sigma(0)=1$, and $\omega=0$ in both (1.3) and (1.4), we obtain the classical left and right-RL fractional integrals (1.1) and (1.2), respectively.

\section{Literature results}

Before we pass to the main findings, we review and introduce some definitions, notations, theorems which will be necessary later to proceed.

Definition 2.1 ([31]) A function $f: \mathcal{I} \subseteq \mathbb{R} \rightarrow \mathbb{R}$ is said to be convex on $\mathcal{I}$ if

$$
f\left((1-\xi) \chi_{1}+\xi \chi_{2}\right) \leq(1-\xi) f\left(\chi_{1}\right)+\xi f\left(\chi_{2}\right)
$$

holds for every $\chi_{1}, \chi_{2} \in \mathcal{I}$ and $\xi \in[0,1]$. 
Definition $2.2([32])$ Denote $\Delta:=\left[\chi_{1}, \chi_{2}\right] \times\left[\chi_{3}, \chi_{4}\right]$, where $0<\chi_{1}<\chi_{2}$ and $0<\chi_{3}<\chi_{4}$. For a function $f: \Delta \rightarrow \mathbb{R}$, the coordinated convex function on $\Delta$ is defined as follows:

$$
\begin{aligned}
& f\left(\left(\xi_{1} \chi_{1}+\left(1-\xi_{1}\right) \chi_{2}\right),\left(\xi_{2} \chi_{3}+\left(1-\xi_{2}\right) \chi_{4}\right)\right) \\
& \leq \xi_{1} \xi_{2} f\left(\chi_{1}, \chi_{3}\right)+\xi_{2}\left(1-\xi_{1}\right) f\left(\chi_{2}, \chi_{3}\right) \\
& \quad+\xi_{1}\left(1-\xi_{2}\right) f\left(\chi_{1}, \chi_{4}\right)+\left(1-\xi_{1}\right)\left(1-\xi_{2}\right) f\left(\chi_{2}, \chi_{4}\right)
\end{aligned}
$$

for every $\xi_{1}, \xi_{2} \in[0,1]$ and $\left(\chi_{1}, \chi_{2}\right),\left(\chi_{3}, \chi_{4}\right) \in \Delta$.

The well-known integral inequality of Hermite-Hadamard type (HH-type) for such a convex function (2.1) is given by

$$
f\left(\frac{\chi_{1}+\chi_{2}}{2}\right) \leq \frac{1}{\chi_{2}-\chi_{1}} \int_{\chi_{1}}^{\chi_{2}} f(x) d x \leq \frac{f\left(\chi_{1}\right)+f\left(\chi_{2}\right)}{2}
$$

In 2001, HH-inequality (2.3) was established on the bidimensional plane $\Delta$ for such a coordinated convex function (2.2) by Dragomir [32], his result is as follows.

Theorem 2.1 Let $f: \Delta \rightarrow \mathbb{R}$ be a coordinated convex function on $\Delta$, then we have

$$
\begin{aligned}
& f\left(\frac{\chi_{1}+\chi_{2}}{2}, \frac{\chi_{3}+\chi_{4}}{2}\right) \\
& \quad \leq \frac{1}{2}\left(\frac{1}{\chi_{2}-\chi_{1}} \int_{\chi_{1}}^{\chi_{2}} f\left(x, \frac{\chi_{3}+\chi_{4}}{2}\right) d x+\frac{1}{\chi_{4}-\chi_{3}} \int_{\chi_{3}}^{\chi_{4}} f\left(\frac{\chi_{1}+\chi_{2}}{2}, y\right) d y\right) \\
& \quad \leq \frac{1}{\left(\chi_{2}-\chi_{1}\right)\left(\chi_{4}-\chi_{3}\right)} \int_{\chi_{1}}^{\chi_{2}} \int_{\chi_{3}}^{\chi_{4}} f(x, y) d y d x \\
& \quad \leq \frac{1}{4}\left(\frac{1}{\chi_{2}-\chi_{1}} \int_{\chi_{1}}^{\chi_{2}}\left(f\left(x, \chi_{3}\right)+f\left(x, \chi_{4}\right)\right) d x \frac{1}{\chi_{4}-\chi_{3}} \int_{\chi_{3}}^{\chi_{4}}\left(f\left(\chi_{1}, y\right)+f\left(\chi_{2}, y\right)\right) d y\right) \\
& \quad \leq \frac{f\left(\chi_{1}, \chi_{3}\right)+f\left(\chi_{1}, \chi_{4}\right)+f\left(\chi_{2}, \chi_{3}\right)+f\left(\chi_{2}, \chi_{4}\right)}{4} .
\end{aligned}
$$

In 2014, HH-inequality (2.4) was generalized to fractional integrals of RL type by Sarikaya [33], which is as follows.

Theorem 2.2 Letf $: \Delta \rightarrow \mathbb{R}$ be a coordinated convex function on $\Delta$, then we have

$$
\begin{aligned}
f( & \left.\frac{\chi_{1}+\chi_{2}}{2}, \frac{\chi_{3}+\chi_{4}}{2}\right) \\
\leq & \frac{\Gamma(\alpha+1)}{4\left(\chi_{2}-\chi_{1}\right)^{\alpha}}\left(J_{\chi_{1}^{+}}^{\alpha} f\left(\chi_{2}, \frac{\chi_{3}+\chi_{4}}{2}\right)+J_{\chi_{2}}^{\alpha} f\left(\chi_{1}, \frac{\chi_{3}+\chi_{4}}{2}\right)\right) \\
& +\frac{\Gamma(\beta+1)}{4\left(\chi_{4}-\chi_{3}\right)^{\beta}}\left(J_{\chi_{3}^{+}}^{\alpha} f\left(\frac{\chi_{1}+\chi_{2}}{2}, \chi_{4}\right)+J_{\chi_{4}^{-}}^{\beta} f\left(\frac{\chi_{1}+\chi_{2}}{2}, \chi_{3}\right)\right) \\
\leq & \frac{\Gamma(\alpha+1) \Gamma(\beta+1)}{\left(\chi_{2}-\chi_{1}\right)^{\alpha}\left(\chi_{4}-\chi_{3}\right)^{\beta}}\left(J_{\chi_{1}^{+}, \chi_{3}^{+}}^{\alpha, \beta} f\left(\chi_{2}, \chi_{4}\right)+J_{\chi_{1}^{+}, \chi_{4}^{-}}^{\alpha, \beta} f\left(\chi_{2}, \chi_{3}\right)\right. \\
& \left.+J_{\chi_{2}^{-}, \chi_{3}^{+}}^{\alpha, \beta} f\left(\chi_{1}, \chi_{4}\right)+J_{\chi_{2}^{2}, \chi_{4}^{-}}^{\alpha, \beta} f\left(\chi_{1}, \chi_{3}\right)\right)
\end{aligned}
$$




$$
\begin{aligned}
\leq & \frac{\Gamma(\alpha+1)}{4\left(\chi_{2}-\chi_{1}\right)^{\alpha}}\left(J_{\chi_{2}^{-}}^{\alpha} f\left(\chi_{1}, \chi_{4}\right)+J_{\chi_{2}^{-}}^{\alpha} f\left(\chi_{1}, \chi_{3}\right)+J_{\chi_{1}^{+}}^{\alpha} f\left(\chi_{2}, \chi_{4}\right)+J_{\chi_{1}^{+}}^{\alpha} f\left(\chi_{2}, \chi_{3}\right)\right) \\
& +\frac{\Gamma(\beta+1)}{4\left(\chi_{4}-\chi_{3}\right)^{\beta}}\left(J_{\chi_{4}^{-}}^{\beta} f\left(\chi_{2}, \chi_{3}\right)+J_{\chi_{4}^{-}}^{\beta} f\left(\chi_{1}, \chi_{3}\right)+J_{\chi_{3}^{+}}^{\alpha} f\left(\chi_{2}, \chi_{4}\right)+J_{\chi_{3}^{+}}^{\alpha} f\left(\chi_{1}, \chi_{4}\right)\right) \\
\leq & \frac{f\left(\chi_{1}, \chi_{3}\right)+f\left(\chi_{1}, \chi_{4}\right)+f\left(\chi_{2}, \chi_{3}\right)+f\left(\chi_{2}, \chi_{4}\right)}{4} .
\end{aligned}
$$

The above inequalities have attracted many researchers in the recent years, see e.g. [3438].

Definition 2.3 ([39]) Let $f \in L(\Delta)$. The fractional integral operators for two variable functions, where $(\rho, \eta, \omega) \in[0,+\infty)^{2} \times[0,+\infty)^{2} \times \mathbb{R}^{2}$ with $\rho=\left(\rho_{1}, \rho_{2}\right), \eta=\left(\eta_{1}, \eta_{2}\right), \omega=\left(\omega_{1}, \omega_{2}\right)$, and $\sigma=\left(\sigma_{1}, \sigma_{2}\right)$, are given as follows:

$$
\begin{aligned}
& \Im_{\rho, \eta, \chi_{1}^{+}, \chi_{3}^{+}, \omega}^{\sigma} \varphi(x, y)=\int_{\chi_{1}}^{x} \int_{\chi_{3}}^{y}\left(x-\xi_{1}\right)^{\eta_{1}-1}\left(y-\xi_{2}\right)^{\eta_{2}-1} \mathfrak{F}_{\rho_{1}, \eta_{1}}^{\sigma_{1}}\left[\omega_{1}\left(x-\xi_{1}\right)^{\rho_{1}}\right] \\
& \times \mathfrak{F}_{\rho_{2}, \eta_{2}}^{\sigma_{2}}\left[\omega_{2}\left(y-\xi_{2}\right)^{\rho_{1}}\right] \varphi\left(\xi_{1}, \xi_{2}\right) d \xi_{2} d \xi_{1}, \\
& \Im_{\rho, \eta, \chi_{1}^{+}, \chi_{4}^{-}, \omega}^{\sigma} \varphi(x, y)=\int_{\chi_{1}}^{x} \int_{y}^{\chi_{4}}\left(x-\xi_{1}\right)^{\eta_{1}-1}\left(\xi_{2}-y\right)^{\eta_{2}-1} \mathfrak{F}_{\rho_{1}, \eta_{1}}^{\sigma_{1}}\left[\omega_{1}\left(x-\xi_{1}\right)^{\rho_{1}}\right] \\
& \times \mathfrak{F}_{\rho_{2}, \eta_{2}}^{\sigma_{2}}\left[\omega_{2}\left(\xi_{2}-y\right)^{\rho_{1}}\right] \varphi\left(\xi_{1}, \xi_{2}\right) d \xi_{2} d \xi_{1} \\
& \Im_{\rho, \eta, \chi_{2}^{-}, \chi_{3}^{+}, \omega}^{\sigma} \varphi(x, y)=\int_{x}^{\chi_{2}} \int_{\chi_{3}}^{y}\left(\xi_{1}-x\right)^{\eta_{1}-1}\left(y-\xi_{2}\right)^{\eta_{2}-1} \mathfrak{F}_{\rho_{1}, \eta_{1}}^{\sigma_{1}}\left[\omega_{1}\left(\xi_{1}-x\right)^{\rho_{1}}\right] \\
& \times \mathfrak{F}_{\rho_{2}, \eta_{2}}^{\sigma_{2}}\left[\omega_{2}\left(y-\xi_{2}\right)^{\rho_{1}}\right] \varphi\left(\xi_{1}, \xi_{2}\right) d \xi_{2} d \xi_{1}
\end{aligned}
$$

and

$$
\begin{aligned}
\Im_{\rho, \eta, \chi_{2}^{-}, \chi_{4}^{-}, \omega}^{\sigma} \varphi(x, y)= & \int_{x}^{\chi_{2}} \int_{y}^{\chi_{4}}\left(\xi_{1}-x\right)^{\eta_{1}-1}\left(\xi_{2}-y\right)^{\eta_{2}-1} \mathfrak{F}_{\rho_{1}, \eta_{1}}^{\sigma_{1}}\left[\omega_{1}\left(\xi_{1}-x\right)^{\rho_{1}}\right] \\
& \times \mathfrak{F}_{\rho_{2}, \eta_{2}}^{\sigma_{2}}\left[\omega_{2}\left(\xi_{2}-y\right)^{\rho_{1}}\right] \varphi\left(\xi_{1}, \xi_{2}\right) d \xi_{2} d \xi_{1} .
\end{aligned}
$$

Also, we have

$$
\begin{aligned}
& \Im_{\rho_{1}, \eta_{1}, \chi_{1}^{+}, \omega_{1}}^{\sigma_{1}} \varphi\left(x, \frac{\chi_{3}+\chi_{4}}{2}\right)=\int_{\chi_{1}}^{x}\left(x-\xi_{1}\right)^{\eta_{1}-1} \mathfrak{F}_{\rho_{1}, \eta_{1}}^{\sigma_{1}}\left[\omega_{1}\left(x-\xi_{1}\right)^{\rho_{1}}\right] \varphi\left(\xi_{1}, \frac{\chi_{3}+\chi_{4}}{2}\right) d \xi_{1}, \\
& \Im_{\rho_{1}, \eta_{1}, \chi_{2}^{-}, \omega_{1}}^{\sigma_{1}} \varphi\left(x, \frac{\chi_{3}+\chi_{4}}{2}\right)=\int_{x}^{\chi_{2}}\left(\xi_{1}-x\right)^{\eta_{1}-1} \mathfrak{F}_{\rho_{1}, \eta_{1}}^{\sigma_{1}}\left[\omega_{1}\left(\xi_{1}-x\right)^{\rho_{1}}\right] \varphi\left(\xi_{1}, \frac{\chi_{3}+\chi_{4}}{2}\right) d \xi_{1}, \\
& \Im_{\rho_{2}, \eta_{2}, \chi_{3}^{+}, \omega_{2}}^{\sigma_{2}} \varphi\left(\frac{\chi_{1}+\chi_{2}}{2}, y\right)=\int_{\chi_{3}}^{y}\left(y-\xi_{2}\right)^{\eta_{2}-1} \mathfrak{F}_{\rho_{2}, \eta_{2}}^{\sigma_{2}}\left[\omega_{2}\left(y-\xi_{2}\right)^{\rho_{1}}\right] \varphi\left(\frac{\chi_{1}+\chi_{2}}{2}, \xi_{2}\right) d \xi_{2},
\end{aligned}
$$

and

$$
\Im_{\rho_{2}, \eta_{2}, \chi_{4}^{-}, \omega_{2}}^{\sigma_{2}} \varphi\left(\frac{\chi_{1}+\chi_{2}}{2}, y\right)=\int_{y}^{\chi_{4}}\left(\xi_{2}-y\right)^{\eta_{2}-1} \mathfrak{F}_{\rho_{2}, \eta_{2}}^{\sigma_{2}}\left[\omega_{2}\left(\xi_{2}-y\right)^{\rho_{1}}\right] \varphi\left(\frac{\chi_{1}+\chi_{2}}{2}, \xi_{2}\right) d \xi_{2} .
$$


In [39], Tunç and Sarikaya investigate the following Hermite-Hadamard for coordinated convex functions:

$$
\begin{aligned}
f( & \left.\frac{\chi_{1}+\chi_{2}}{2}, \frac{\chi_{3}+\chi_{4}}{2}\right) \\
\leq & \frac{1}{\left(\chi_{2}-\chi_{1}\right)^{\eta_{1}}\left(\chi_{4}-\chi_{3}\right)^{\eta_{2}} \mathfrak{F}_{\rho_{1}, \eta_{1}+1}^{\sigma_{1}}\left(\omega_{1}\left(\chi_{2}-\chi_{1}\right)^{\rho_{1}}\right) \mathfrak{F}_{\rho_{2}, \eta_{2}+1}^{\sigma_{2}}\left(\omega_{2}\left(\chi_{4}-\chi_{3}\right)^{\rho_{2}}\right)} \\
& \times\left\{\Im_{\rho, \eta, \chi_{1}^{+}, \chi_{3}^{+}, \omega}^{\sigma} f\left(\chi_{2}, \chi_{4}\right)+\Im_{\rho, \eta, \chi_{1}^{+}, \chi_{4}^{-}, \omega}^{\sigma} f\left(\chi_{2}, \chi_{3}\right)\right. \\
& \left.+\Im_{\rho, \eta, \chi_{2}^{-}, \chi_{3}^{+}, \omega}^{\sigma} f\left(\chi_{1}, \chi_{4}\right)+\Im_{\rho, \eta, \chi_{2}^{-}, \chi_{4}^{-}, \omega}^{\sigma} f\left(\chi_{1}, \chi_{3}\right)\right\} \\
\leq & \frac{\left(f\left(\chi_{1}, \chi_{3}\right)+f\left(\chi_{1}, \chi_{4}\right)+f\left(\chi_{2}, \chi_{3}\right)+f\left(\chi_{2}, \chi_{4}\right)\right)}{4} .
\end{aligned}
$$

Definition 2.4 ([40]) Let $h_{1}, h_{2}: J \rightarrow \mathbb{R}$ be two nonnegative and nonzero functions. A function $f: \Delta \rightarrow \mathbb{R}$ is said to be $\left(l_{1}, h_{1}\right)-\left(l_{2}, h_{2}\right)$-convex function on the coordinates on $\Delta$ if

$$
\begin{aligned}
f\left(\left[\xi_{1} x^{l_{1}}+\left(1-\xi_{1}\right) u^{l_{1}}\right]^{\frac{1}{l_{1}}},\left[\xi_{2} y^{l_{2}}+\left(1-\xi_{2}\right) v^{l_{2}}\right]^{\frac{1}{l_{2}}}\right) \\
\quad \leq h_{1}\left(\xi_{1}\right) h_{2}\left(\xi_{2}\right) f(x, y)+h_{1}\left(\xi_{1}\right) h_{2}\left(1-\xi_{2}\right) f(x, v)+h_{1}\left(1-\xi_{1}\right) h_{2}\left(\xi_{2}\right) f(u, y) \\
\quad+h_{1}\left(1-\xi_{1}\right) h_{2}\left(1-\xi_{2}\right) f(u, v)
\end{aligned}
$$

holds for all $(x, y),(u, v) \in \Delta$ and $\xi_{1}, \xi_{2} \in(0,1)$

As we know, there are many results on coordinates of convex functions via other types of fractional operators and other types of convex functions, see e.g. [41-44]. Therefore, integral inequalities on coordinates of convex functions via general Raina fractional integrals open a new door in the field of mathematical analysis and theory of convexity.

Motivated by the above results, in this paper we establish some generalized integral inequalities using an $\left(l_{1}, h_{1}\right)-\left(l_{2}, h_{2}\right)$-convex function on coordinates. Also, we obtain an integral identity for partial differentiable functions. As an effect of this result, two interesting integral inequalities for an $\left(l_{1}, h_{1}\right)-\left(l_{2}, h_{2}\right)$-convex function on coordinates are given. At the end, a brief conclusion is provided as well.

\section{Main results}

In what follows, we assume that $h_{1}, h_{2}: J \rightarrow \mathbb{R}$ are two nonnegative and nonzero functions, with $h_{1}\left(\frac{1}{2}\right) h_{2}\left(\frac{1}{2}\right) \neq 0, \sigma=\left(\sigma_{1}, \sigma_{2}\right), \rho=\left(\rho_{1}, \rho_{2}\right), \eta=\left(\eta_{1}, \eta_{2}\right)$, and $\omega=\left(\omega_{1}, \omega_{2}\right)$ with $\rho_{1}, \rho_{2}, \eta_{1}, \eta_{2} \in[0,+\infty)$ and $\omega_{1}, \omega_{2} \in \mathbb{R}$.

Theorem 3.1 Let $f: \Delta \rightarrow \mathbb{R}$ be an integrable and $\left(l_{1}, h_{1}\right)-\left(l_{2}, h_{2}\right)$-convex function on coordinates on $\Delta$. Then we have

$$
\begin{gathered}
\frac{1}{h_{1}\left(\frac{1}{2}\right) h_{2}\left(\frac{1}{2}\right)} f\left(\left[\frac{\chi_{1}^{l_{1}}+\chi_{2}^{l_{1}}}{2}\right]^{\frac{1}{l_{1}}},\left[\frac{\chi_{3}^{l_{2}}+\chi_{4}^{l_{2}}}{2}\right]^{\frac{1}{l_{2}}}\right) \\
=\frac{1}{h_{1}\left(\frac{1}{2}\right) h_{2}\left(\frac{1}{2}\right)} f_{g}\left(\frac{\chi_{1}^{l_{1}}+\chi_{2}^{l_{1}}}{2}, \frac{\chi_{3}^{l_{2}}+\chi_{4}^{l_{2}}}{2}\right)
\end{gathered}
$$




$$
\begin{aligned}
& \leq \frac{1}{\left(\chi_{2}^{l_{1}}-\chi_{1}^{l_{1}}\right)^{\eta_{1}}\left(\chi_{4}^{l_{2}}-\chi_{3}^{l_{2}}\right)^{\eta_{2}} \mathfrak{F}_{\rho_{1}, \eta_{1}+1}^{\sigma_{1}}\left(\omega_{1}\left(\chi_{2}^{l_{1}}-\chi_{1}^{l_{1}}\right)^{\rho_{1}}\right) \mathfrak{F}_{\rho_{2}, \eta_{2}+1}^{\sigma_{2}}\left(\omega_{2}\left(\chi_{4}^{l_{2}}-\chi_{3}^{l_{2}}\right)^{\rho_{2}}\right)} \\
& \times\left\{\Im_{\rho, \eta,\left(\chi_{1}^{l_{1}}\right)^{+},\left(\chi_{3}^{l_{2}}\right)^{+}, \omega} f_{g}\left(\chi_{2}^{l_{1}}, \chi_{4}^{l_{2}}\right)+\Im_{\rho, \eta,\left(\chi_{1}^{l_{1}}\right)^{+},\left(\chi_{4}^{l_{2}}\right)^{-}, \omega}^{\sigma} f_{g}\left(\chi_{2}^{l_{1}}, \chi_{3}^{l_{2}}\right)\right. \\
& \left.+\Im_{\rho, \eta,\left(\chi_{2}^{l_{1}}\right)^{-},\left(\chi_{3}^{l_{2}}\right)^{+}, \omega}^{\sigma} f_{g}\left(\chi_{1}^{l_{1}}, \chi_{4}^{l_{2}}\right)+\Im_{\rho, \eta,\left(\chi_{2}^{l_{1}}\right)^{-},\left(\chi_{4}^{l_{2}}\right)^{-}, \omega}^{\sigma} f_{g}\left(\chi_{1}^{l_{1}}, \chi_{3}^{l_{2}}\right)\right\} \\
& \leq \frac{\left(f\left(\chi_{1}, \chi_{3}\right)+f\left(\chi_{1}, \chi_{4}\right)+f\left(\chi_{2}, \chi_{3}\right)+f\left(\chi_{2}, \chi_{4}\right)\right)}{\mathfrak{F}_{\rho_{1}, \eta_{1}+1}^{\sigma_{1}}\left(\omega_{1}\left(\chi_{2}^{l_{1}}-\chi_{1}^{l_{1}}\right)^{\rho_{1}}\right) \mathfrak{F}_{\rho_{2}, \eta_{2}+1}^{\sigma_{2}}\left(\omega_{2}\left(\chi_{4}^{l_{2}}-\chi_{3}^{l_{2}}\right)^{\rho_{2}}\right)} \\
& \times \int_{0}^{1} \int_{0}^{1} \xi_{1}^{\eta_{1}-1} \xi_{2}^{\eta_{2}-1} \mathfrak{F}_{\rho_{1}, \eta_{1}}^{\sigma_{1}}\left[\omega_{1}\left(\chi_{2}^{l_{1}}-\chi_{1}^{l_{1}}\right)^{\rho_{1}} \xi_{1}^{\rho_{1}}\right] \mathfrak{F}_{\rho_{2}, \eta_{2}}^{\sigma_{2}}\left[\omega_{2}\left(\chi_{4}^{l_{2}}-\chi_{3}^{l_{2}}\right)^{\rho_{2}} \xi_{2}^{\rho_{2}}\right] \\
& \times\left(h_{1}\left(\xi_{1}\right)+h_{1}\left(1-\xi_{1}\right)\right)\left(h_{2}\left(\xi_{2}\right)+h_{2}\left(1-\xi_{2}\right)\right) d \xi_{2} d \xi_{1},
\end{aligned}
$$

where $f_{g}(x, y)=f\left(g_{1}(x), g_{2}(y)\right)$ with $g_{1}(x)=x^{\frac{1}{l_{1}}}$ and $g_{2}(y)=y^{\frac{1}{l_{2}}}$.

Proof It is easy to see that

$$
\left[\frac{\chi_{1}^{l_{1}}+\chi_{2}^{l_{1}}}{2}\right]^{\frac{1}{l_{1}}}=\left[\frac{\left(\left(\xi_{1} \chi_{1}^{l_{1}}+\left(1-\xi_{1}\right) \chi_{2}^{l_{1}}\right)^{\frac{1}{l_{1}}}\right)^{l_{1}}+\left(\left(\left(1-\xi_{1}\right) \chi_{1}^{l_{1}}+\xi_{1} \chi_{2}^{l_{1}}\right)^{\frac{1}{l_{1}}}\right)^{l_{1}}}{2}\right]^{\frac{1}{l_{1}}}
$$

and

$$
\left[\frac{\chi_{3}^{l_{2}}+\chi_{4}^{l_{2}}}{2}\right]^{\frac{1}{l_{2}}}=\left[\frac{\left(\left(\xi_{2} \chi_{3}^{l_{2}}+\left(1-\xi_{2}\right) \chi_{4}^{l_{2}}\right)^{\frac{1}{l_{2}}}\right)^{l_{2}}+\left(\left(\left(1-\xi_{2}\right) \chi_{3}^{l_{2}}+\xi_{2} \chi_{4}^{l_{2}}\right)^{\frac{1}{l_{2}}}\right)^{l_{2}}}{2}\right]^{\frac{1}{l_{2}}} .
$$

Making use of (3.1) and (3.2), and the fact that $f$ is $\left(l_{1}, h_{1}\right)-\left(l_{2}, h_{2}\right)$-convex on the coordinates, we have

$$
\begin{aligned}
& f\left(\left[\frac{\chi_{1}^{l_{1}}+\chi_{2}^{l_{1}}}{2}\right]^{\frac{1}{l_{1}}},\left[\frac{\chi_{3}^{l_{2}}+\chi_{4}^{l_{2}}}{2}\right]^{\frac{1}{l_{2}}}\right) \\
& \leq h_{1}\left(\frac{1}{2}\right) h_{2}\left(\frac{1}{2}\right)\left\{f\left(\left(\xi_{1} \chi_{1}^{l_{1}}+\left(1-\xi_{1}\right) \chi_{2}^{l_{1}}\right)^{\frac{1}{l_{1}}},\left(\xi_{2} \chi_{3}^{l_{2}}+\left(1-\xi_{2}\right) \chi_{4}^{l_{2}}\right)^{\frac{1}{l_{2}}}\right)\right. \\
&+f\left(\left(\xi_{1} \chi_{1}^{l_{1}}+\left(1-\xi_{1}\right) \chi_{2}^{l_{1}}\right)^{\frac{1}{l_{1}}},\left(\left(1-\xi_{2}\right) \chi_{3}^{l_{2}}+\xi_{2} \chi_{4}^{l_{2}}\right)^{\frac{1}{l_{2}}}\right) \\
&+f\left(\left(\left(1-\xi_{1}\right) \chi_{1}^{l_{1}}+\xi_{1} \chi_{2}^{l_{1}}\right)^{\frac{1}{l_{1}}},\left(\xi_{2} \chi_{3}^{l_{2}}+\left(1-\xi_{2}\right) \chi_{4}^{l_{2}}\right)^{\frac{1}{l_{2}}}\right) \\
&\left.+f\left(\left(\left(1-\xi_{1}\right) \chi_{1}^{l_{1}}+\xi_{1} \chi_{2}^{l_{1}}\right)^{\frac{1}{l_{1}}},\left(\left(1-\xi_{2}\right) \chi_{3}^{l_{2}}+\xi_{2} \chi_{4}^{l_{2}}\right)^{\frac{1}{l_{2}}}\right)\right\} .
\end{aligned}
$$

Multiplying on both sides of (3.3) by

$$
\xi_{1}^{\eta_{1}-1} \xi_{2}^{\eta_{2}-1} \mathfrak{F}_{\rho_{1}, \eta_{1}}^{\sigma_{1}}\left[\omega_{1}\left(\chi_{2}^{l_{1}}-\chi_{1}^{l_{1}}\right)^{\rho_{1}} \xi_{1}^{\rho_{1}}\right] \mathfrak{F}_{\rho_{2}, \eta_{2}}^{\sigma_{2}}\left[\omega_{2}\left(\chi_{4}^{l_{2}}-\chi_{3}^{l_{2}}\right)^{\rho_{2}} \xi_{2}^{\rho_{2}}\right]
$$

and then integrating the resulting inequality with respect to $\left(\xi_{1}, \xi_{2}\right)$ on $[0,1]^{2}$, we get

$$
\begin{aligned}
& \frac{1}{h_{1}\left(\frac{1}{2}\right) h_{2}\left(\frac{1}{2}\right)} f\left(\left[\frac{\chi_{1}^{l_{1}}+\chi_{2}^{l_{1}}}{2}\right]^{\frac{1}{l_{1}}},\left[\frac{\chi_{3}^{l_{2}}+\chi_{4}^{l_{2}}}{2}\right]^{\frac{1}{l_{2}}}\right) \\
& \quad \times \int_{0}^{1} \int_{0}^{1} \xi_{1}^{\eta_{1}-1} \xi_{2}^{\eta_{2}-1} \mathfrak{F}_{\rho_{1}, \eta_{1}}^{\sigma_{1}}\left[\omega_{1}\left(\chi_{2}^{l_{1}}-\chi_{1}^{l_{1}}\right)^{\rho_{1}} \xi_{1}^{\rho_{1}}\right] \mathfrak{F}_{\rho_{2}, \eta_{2}}^{\sigma_{2}}\left[\omega_{2}\left(\chi_{4}^{l_{2}}-\chi_{3}^{l_{2}}\right)^{\rho_{2}} \xi_{2}^{\rho_{2}}\right] d \xi_{2} d \xi_{1}
\end{aligned}
$$




$$
\begin{aligned}
\leq & \int_{0}^{1} \int_{0}^{1} \xi_{1}^{\eta_{1}-1} \xi_{2}^{\eta_{2}-1} \mathfrak{F}_{\rho_{1}, \eta_{1}}^{\sigma_{1}}\left[\omega_{1}\left(\chi_{2}^{l_{1}}-\chi_{1}^{l_{1}}\right)^{\rho_{1}} \xi_{1}^{\rho_{1}}\right] \mathfrak{F}_{\rho_{2}, \eta_{2}}^{\sigma_{2}}\left[\omega_{2}\left(\chi_{4}^{l_{2}}-\chi_{3}^{l_{2}}\right)^{\rho_{2}} \xi_{2}^{\rho_{2}}\right] \\
& \times f\left(\left[\xi_{1} \chi_{1}^{l_{1}}+\left(1-\xi_{1}\right) \chi_{2}^{l_{1}}\right]^{\frac{1}{l_{1}}},\left[\xi_{2} \chi_{3}^{l_{2}}+\left(1-\xi_{2}\right) \chi_{4}^{l_{2}}\right]^{\frac{1}{l_{2}}}\right) d \xi_{2} d \xi_{1} \\
& +\int_{0}^{1} \int_{0}^{1} \xi_{1}^{\eta_{1}-1} \xi_{2}^{\eta_{2}-1} \mathfrak{F}_{\rho_{1}, \eta_{1}}^{\sigma_{1}}\left[\omega_{1}\left(\chi_{2}^{l_{1}}-\chi_{1}^{l_{1}}\right)^{\rho_{1}} \xi_{1}^{\rho_{1}}\right] \mathfrak{F}_{\rho_{2}, \eta_{2}}^{\sigma_{2}}\left[\omega_{2}\left(\chi_{4}^{l_{2}}-\chi_{3}^{l_{2}}\right)^{\rho_{2}} \xi_{2}^{\rho_{2}}\right] \\
& \times f\left(\left[\xi_{1} \chi_{1}^{l_{1}}+\left(1-\xi_{1}\right) \chi_{2}^{l_{1}}\right]^{\frac{1}{l_{1}}},\left[\left(1-\xi_{2}\right) \chi_{3}^{l_{2}}+\xi_{2} \chi_{4}^{l_{2}}\right]^{\frac{1}{l_{2}}}\right) d \xi_{2} d \xi_{1} \\
& +\int_{0}^{1} \int_{0}^{1} \xi_{1}^{\eta_{1}-1} \xi_{2}^{\eta_{2}-1} \mathfrak{F}_{\rho_{1}, \eta_{1}}^{\sigma_{1}}\left[\omega_{1}\left(\chi_{2}^{l_{1}}-\chi_{1}^{l_{1}}\right)^{\rho_{1}} \xi_{1}^{\rho_{1}}\right] \mathfrak{F}_{\rho_{2}, \eta_{2}}^{\sigma_{2}}\left[\omega_{2}^{\rho_{2}}\left(\chi_{4}^{l_{2}}-\chi_{3}^{l_{2}}\right) \xi_{2}^{\rho_{2}}\right] \\
& \times f\left(\left[\left(1-\xi_{1}\right) \chi_{1}^{l_{1}}+\xi_{1} \chi_{2}^{l_{1}}\right]^{\frac{1}{l_{1}}},\left[\xi_{2} \chi_{3}^{l_{2}}+\left(1-\xi_{2}\right) \chi_{4}^{l_{2}}\right]^{\frac{1}{2}}\right) d \xi_{2} d \xi_{1} \\
& +\int_{0}^{1} \int_{0}^{1} \xi_{1}^{\eta_{1}-1} \xi_{2}^{\eta_{2}-1} \mathfrak{F}_{\rho_{1}, \eta_{1}}^{\sigma_{1}}\left[\omega_{1}\left(\chi_{2}^{l_{1}}-\chi_{1}^{l_{1}}\right)^{\rho_{1}} \xi_{1}^{\rho_{1}}\right] \mathfrak{F}_{\rho_{2}, \eta_{2}}^{\sigma_{2}}\left[\omega_{2}\left(\chi_{4}^{l_{2}}-\chi_{3}^{l_{2}}\right)^{\rho_{2}} \xi_{2}^{\rho_{2}}\right] \\
& \times f\left(\left[\left(1-\xi_{1}\right) \chi_{1}^{l_{1}}+\xi_{1} \chi_{2}^{l_{1}}\right]^{\frac{1}{l_{1}}},\left[\left(1-\xi_{2}\right) \chi_{3}^{l_{2}}+\xi_{2} \chi_{4}^{l_{2}}\right]^{\frac{1}{l_{2}}}\right) d \xi_{2} d \xi_{1} .
\end{aligned}
$$

By making a change of variables in (3.4), we obtain

$$
\begin{aligned}
& \frac{1}{h_{1}\left(\frac{1}{2}\right) h_{2}\left(\frac{1}{2}\right)} f\left(\left[\frac{\chi_{1}^{l_{1}}+\chi_{2}^{l_{1}}}{2}\right]^{\frac{1}{l_{1}}},\left[\frac{\chi_{3}^{l_{2}}+\chi_{4}^{l_{2}}}{2}\right]^{\frac{1}{l_{2}}}\right) \\
& \leq \frac{1}{\left(\chi_{2}^{l_{1}}-\chi_{1}^{l_{1}}\right)^{\eta_{1}}\left(\chi_{4}^{l_{2}}-\chi_{3}^{l_{2}}\right)^{\eta_{2}} \mathfrak{F}_{\rho_{1}, \eta_{1}+1}^{\sigma_{1}}\left(\omega_{1}\left(\chi_{2}^{l_{1}}-\chi_{1}^{l_{1}}\right)^{\rho_{1}}\right) \mathfrak{F}_{\rho_{2}, \eta_{2}+1}^{\sigma_{2}}\left(\omega_{2}\left(\chi_{4}^{l_{2}}-\chi_{3}^{l_{2}}\right)^{\rho_{2}}\right)} \\
& \times\left\{\int_{\chi_{1}^{l_{1}}}^{\chi_{2}^{l_{1}}} \int_{\chi_{3}^{l_{2}}}^{\chi_{4}^{l_{2}}}\left(\chi_{2}^{l_{1}}-x\right)^{\eta_{1}-1}\left(\chi_{4}^{l_{2}}-y\right)^{\eta_{2}-1} \mathfrak{F}_{\rho_{1}, \eta_{1}}^{\sigma_{1}}\left[\omega_{1}\left(\chi_{2}^{l_{1}}-x\right)^{\rho_{1}}\right]\right. \\
& \times \mathfrak{F}_{\rho_{2}, \eta_{2}}^{\sigma_{2}}\left[\omega_{2}\left(\chi_{4}^{l_{2}}-y\right)^{\rho_{2}}\right] f\left(x^{\frac{1}{l_{1}}}, y^{\frac{1}{l_{2}}}\right) d y d x \\
& +\int_{\chi_{1}^{l_{1}}}^{\chi_{2}^{l_{1}}} \int_{\chi_{3}^{l_{2}}}^{\chi_{4}^{l_{2}}}\left(\chi_{2}^{l_{1}}-x\right)^{\eta_{1}-1}\left(y-\chi_{3}^{l_{2}}\right)^{\eta_{2}-1} \mathfrak{F}_{\rho_{1}, \eta_{1}}^{\sigma_{1}}\left[\omega_{1}\left(\chi_{2}^{l_{1}}-x\right)^{\rho_{1}}\right] \\
& \times \mathfrak{F}_{\rho_{2}, \eta_{2}}^{\sigma_{2}}\left[\omega_{2}\left(y-\chi_{3}^{l_{2}}\right)^{\rho_{2}}\right] f\left(x^{\frac{1}{l_{1}}}, y^{\frac{1}{l_{2}}}\right) d y d x \\
& +\int_{\chi_{1}^{l_{1}}}^{\chi_{2}^{l_{1}}} \int_{\chi_{3}^{l_{2}}}^{\chi_{4}^{l_{2}}}\left(x-\chi_{1}^{l_{1}}\right)^{\eta_{1}-1}\left(\chi_{4}^{l_{2}}-y\right)^{\eta_{2}-1} \mathfrak{F}_{\rho_{1}, \eta_{1}}^{\sigma_{1}}\left[\omega_{1}\left(x-\chi_{1}^{l_{1}}\right)^{\rho_{1}}\right] \\
& \times \mathfrak{F}_{\rho_{2}, \eta_{2}}^{\sigma_{2}}\left[\omega_{2}^{\rho_{2}}\left(\chi_{4}^{l_{2}}-y\right)\right] f\left(x^{\frac{1}{l_{1}}}, y^{\frac{1}{l_{2}}}\right) d y d x \\
& +\int_{\chi_{1}^{l_{1}}}^{\chi_{2}^{l_{1}}} \int_{\chi_{3}^{l_{2}}}^{\chi_{4}^{l_{2}}}\left(x-\chi_{1}^{l_{1}}\right)^{\eta_{1}-1}\left(y-\chi_{3}^{l_{2}}\right)^{\eta_{2}-1} \mathfrak{F}_{\rho_{1}, \eta_{1}}^{\sigma_{1}}\left[\omega_{1}\left(x-\chi_{1}^{l_{1}}\right)^{\rho_{1}}\right] \\
& \left.\times \mathfrak{F}_{\rho_{2}, \eta_{2}}^{\sigma_{2}}\left[\omega_{2}\left(y-\chi_{3}^{l_{2}}\right)^{\rho_{2}}\right] f\left(x^{\frac{1}{l_{1}}}, y^{\frac{1}{l_{2}}}\right) d y d x\right\} \\
& =\frac{1}{\left(\chi_{2}^{l_{1}}-\chi_{1}^{l_{1}}\right)^{\eta_{1}}\left(\chi_{4}^{l_{2}}-\chi_{3}^{l_{2}}\right)^{\eta_{2}} \mathfrak{F}_{\rho_{1}, \eta_{1}+1}^{\sigma_{1}}\left(\omega_{1}\left(\chi_{2}^{l_{1}}-\chi_{1}^{l_{1}}\right)^{\rho_{1}}\right) \mathfrak{F}_{\rho_{2}, \eta_{2}+1}^{\sigma_{2}}\left(\omega_{2}\left(\chi_{4}^{l_{2}}-\chi_{3}^{l_{2}}\right)^{\rho_{2}}\right)} \\
& \times\left\{\Im_{\rho, \eta,\left(\chi_{1}^{l_{1}}\right)^{+},\left(\chi_{3}^{l_{2}}\right)^{+}, \omega} f_{g}\left(\chi_{2}^{l_{1}}, \chi_{4}^{l_{2}}\right)+\Im_{\rho, \eta,\left(\chi_{1}^{l_{1}}\right)^{+},\left(\chi_{4}^{l_{2}}\right)^{-}, \omega}^{\sigma} f_{g}\left(\chi_{2}^{l_{1}}, \chi_{3}^{l_{2}}\right)\right. \\
& \left.+\Im_{\rho, \eta,\left(\chi_{2}^{l_{1}}\right)^{-},\left(\chi_{3}^{l_{2}}\right)^{+}, \omega}^{\sigma} f_{g}\left(\chi_{1}^{l_{1}}, \chi_{4}^{l_{2}}\right)+\Im_{\rho, \eta,\left(\chi_{2}^{l_{1}}\right)^{-},\left(\chi_{4}^{l_{2}}\right)^{-},{ }^{\prime}, f_{g}}^{\sigma}\left(\chi_{1}^{l_{1}}, \chi_{3}^{l_{2}}\right)\right\} .
\end{aligned}
$$


Since $f$ is $\left(l_{1}, h_{1}\right)-\left(l_{2}, h_{2}\right)$-convex on the coordinates, we have

$$
\begin{aligned}
& f\left(\left[\xi_{1} \chi_{1}^{l_{1}}+\left(1-\xi_{1}\right) \chi_{2}^{l_{1}}\right]^{\frac{1}{l_{1}}},\left[\xi_{2} \chi_{3}^{l_{2}}+\left(1-\xi_{2}\right) \chi_{4}^{l_{2}}\right]^{\frac{1}{l_{2}}}\right) \\
& \leq h_{1}\left(\xi_{1}\right) h_{2}\left(\xi_{2}\right) f\left(\chi_{1}, \chi_{3}\right)+h_{1}\left(\xi_{1}\right) h_{2}\left(1-\xi_{2}\right) f\left(\chi_{1}, \chi_{4}\right)+h_{1}\left(1-\xi_{1}\right) h_{2}\left(\xi_{2}\right) f\left(\chi_{2}, \chi_{3}\right) \\
& +h_{1}\left(1-\xi_{1}\right) h_{2}\left(1-\xi_{2}\right) f\left(\chi_{2}, \chi_{4}\right) \text {, } \\
& f\left(\left[\xi_{1} \chi_{1}^{l_{1}}+\left(1-\xi_{1}\right) \chi_{2}^{l_{1}}\right]^{\frac{1}{l_{1}}},\left[\left(1-\xi_{2}\right) \chi_{3}^{l_{2}}+\xi_{2} \chi_{4}^{l_{2}}\right]^{\frac{1}{l_{2}}}\right) \\
& \leq h_{1}\left(\xi_{1}\right) h_{2}\left(1-\xi_{2}\right) f\left(\chi_{1}, \chi_{3}\right)+h_{1}\left(\xi_{1}\right) h_{2}\left(\xi_{2}\right) f\left(\chi_{1}, \chi_{4}\right)+h_{1}\left(1-\xi_{1}\right) h_{2}\left(1-\xi_{2}\right) f\left(\chi_{2}, \chi_{3}\right) \\
& +h_{1}\left(1-\xi_{1}\right) h_{2}\left(\xi_{2}\right) f\left(\chi_{2}, \chi_{4}\right) \text {, } \\
& f\left(\left(\left(1-\xi_{1}\right) \chi_{1}^{l_{1}}+\xi_{1} \chi_{2}^{l_{1}}\right)^{\frac{1}{l_{1}}},\left(\xi_{2} \chi_{3}^{l_{2}}+\left(1-\xi_{2}\right) \chi_{4}^{l_{2}}\right)^{\frac{1}{l_{2}}}\right) \\
& \leq h_{1}\left(1-\xi_{1}\right) h_{2}\left(\xi_{2}\right) f\left(\chi_{1}, \chi_{3}\right)+h_{1}\left(1-\xi_{1}\right) h_{2}\left(1-\xi_{2}\right) f\left(\chi_{1}, \chi_{4}\right)+h_{1}\left(\xi_{1}\right) h_{2}\left(\xi_{2}\right) f\left(\chi_{2}, \chi_{3}\right) \\
& +h_{1}\left(\xi_{1}\right) h_{2}\left(1-\xi_{2}\right) f\left(\chi_{2}, \chi_{4}\right) \text {, }
\end{aligned}
$$

and

$$
\begin{aligned}
f\left(\left(\left(1-\xi_{1}\right) \chi_{1}^{l_{1}}+\xi_{1} \chi_{2}^{l_{1}}\right)^{\frac{1}{l_{1}}},\left(\left(1-\xi_{2}\right) \chi_{3}^{l_{2}}+\xi_{2} \chi_{4}^{l_{2}}\right)^{\frac{1}{l_{2}}}\right) \\
\leq h_{1}\left(1-\xi_{1}\right) h_{2}\left(1-\xi_{2}\right) f\left(\chi_{1}, \chi_{3}\right)+h_{1}\left(1-\xi_{1}\right) h_{2}\left(\xi_{2}\right) f\left(\chi_{1}, \chi_{4}\right) \\
\quad+h_{1}\left(\xi_{1}\right) h_{2}\left(1-\xi_{2}\right) f\left(\chi_{2}, \chi_{3}\right)+h_{1}\left(\xi_{1}\right) h_{2}\left(\xi_{2}\right) f\left(\chi_{2}, \chi_{4}\right) .
\end{aligned}
$$

Adding inequalities (3.6)-(3.9), multiplying the resulting inequality by

$$
\xi_{1}^{\eta_{1}-1} \xi_{2}^{\eta_{2}-1} \mathfrak{F}_{\rho_{1}, \eta_{1}}^{\sigma_{1}}\left[\omega_{1}\left(\chi_{2}^{l_{1}}-\chi_{1}^{l_{1}}\right)^{\rho_{1}} \xi_{1}^{\rho_{1}}\right] \mathfrak{F}_{\rho_{2}, \eta_{2}}^{\sigma_{2}}\left[\omega_{2}\left(\chi_{4}^{l_{2}}-\chi_{3}^{l_{2}}\right)^{\rho_{2}} \xi_{2}^{\rho_{2}}\right]
$$

and then integrating the result with respect to $\left(\xi_{1}, \xi_{2}\right)$ on $[0,1]^{2}$, we get

$$
\begin{aligned}
& \int_{0}^{1} \int_{0}^{1} \xi_{1}^{\eta_{1}-1} \xi_{2}^{\eta_{2}-1} \mathfrak{F}_{\rho_{1}, \eta_{1}}^{\sigma_{1}}\left[\omega_{1}\left(\chi_{2}^{l_{1}}-\chi_{1}^{l_{1}}\right)^{\rho_{1}} \xi_{1}^{\rho_{1}}\right] \mathfrak{F}_{\rho_{2}, \eta_{2}}^{\sigma_{2}}\left[\omega_{2}\left(\chi_{4}^{l_{2}}-\chi_{3}^{l_{2}}\right)^{\rho_{2}} \xi_{2}^{\rho_{2}}\right] \\
& \quad \times\left\{f\left(\left[\xi_{1} \chi_{1}^{l_{1}}+\left(1-\xi_{1}\right) \chi_{2}^{l_{1}}\right]^{\frac{1}{l_{1}}},\left[\xi_{2} \chi_{3}^{l_{2}}+\left(1-\xi_{2}\right) \chi_{4}^{l_{2}}\right]^{\frac{1}{l_{2}}}\right)\right. \\
& \quad+f\left(\left[\xi_{1} \chi_{1}^{l_{1}}+\left(1-\xi_{1}\right) \chi_{2}^{l_{1}}\right]^{\frac{1}{l_{1}}},\left[\left(1-\xi_{2}\right) \chi_{3}^{l_{2}}+\xi_{2} \chi_{4}^{l_{2}}\right]^{\frac{1}{l_{2}}}\right) \\
& \quad+f\left(\left(\left(1-\xi_{1}\right) \chi_{1}^{l_{1}}+\xi_{1} \chi_{2}^{l_{1}}\right)^{\frac{1}{l_{1}}},\left(\xi_{2} \chi_{3}^{l_{2}}+\left(1-\xi_{2}\right) \chi_{4}^{l_{2}}\right)^{\frac{1}{l_{2}}}\right) \\
&\left.\quad+f\left(\left(\left(1-\xi_{1}\right) \chi_{1}^{l_{1}}+\xi_{1} \chi_{2}^{l_{1}}\right)^{\frac{1}{l_{1}}},\left(\left(1-\xi_{2}\right) \chi_{3}^{l_{2}}+\xi_{2} \chi_{4}^{l_{2}}\right)^{\frac{1}{l_{2}}}\right)\right\} d \xi_{2} d \xi_{1} \\
& \leq\left.f\left(\chi_{1}, \chi_{3}\right)+f\left(\chi_{1}, \chi_{4}\right)+f\left(\chi_{2}, \chi_{3}\right)+f\left(\chi_{2}, \chi_{4}\right)\right) \\
& \times \int_{0}^{1} \int_{0}^{1} \xi_{1}^{\eta_{1}-1} \xi_{2}^{\eta_{2}-1} \mathfrak{F}_{\rho_{1}, \eta_{1}}^{\sigma_{1}}\left[\omega_{1}\left(\chi_{2}^{l_{1}}-\chi_{1}^{l_{1}}\right)^{\rho_{1}} \xi_{1}^{\rho_{1}}\right] \mathfrak{F}_{\rho_{2}, \eta_{2}}^{\sigma_{2}}\left[\omega_{2}\left(\chi_{4}^{l_{2}}-\chi_{3}^{l_{2}}\right)^{\rho_{2}} \xi_{2}^{\rho_{2}}\right] \\
& \times\left(h_{1}\left(\xi_{1}\right) h_{2}\left(\xi_{2}\right)+h_{1}\left(\xi_{1}\right) h_{2}\left(1-\xi_{2}\right)+h_{1}\left(1-\xi_{1}\right) h_{2}\left(\xi_{2}\right)\right. \\
&\left.+h_{1}\left(1-\xi_{1}\right) h_{2}\left(1-\xi_{2}\right)\right) d \xi_{2} d \xi_{1} .
\end{aligned}
$$


Making use of the change of variables and multiplying the result by

$$
\frac{1}{\mathfrak{F}_{\rho_{1}, \eta_{1}+1}^{\sigma_{1}}\left(\omega_{1}\left(\chi_{2}^{l_{1}}-\chi_{1}^{l_{1}}\right)^{\rho_{1}}\right) \mathfrak{F}_{\rho_{2}, \eta_{2}+1}^{\sigma_{2}}\left(\omega_{2}\left(\chi_{4}^{l_{2}}-\chi_{3}^{l_{2}}\right)^{\rho_{2}}\right)},
$$

we obtain

$$
\begin{aligned}
& \frac{1}{\left(\chi_{2}^{l_{1}}-\chi_{1}^{l_{1}}\right)^{\eta_{1}}\left(\chi_{4}^{l_{2}}-\chi_{3}^{l_{2}}\right)^{\eta_{2}} \mathfrak{F}_{\rho_{1}, \eta_{1}+1}^{\sigma_{1}}\left(\omega_{1}\left(\chi_{2}^{l_{1}}-\chi_{1}^{l_{1}}\right)^{\rho_{1}}\right) \mathfrak{F}_{\rho_{2}, \eta_{2}+1}^{\sigma_{2}}\left(\omega_{2}\left(\chi_{4}^{l_{2}}-\chi_{3}^{l_{2}}\right)^{\rho_{2}}\right)} \\
& \times\left\{\Im_{\rho, \eta,\left(\chi_{1}^{\sigma}\right)^{+},\left(\chi_{3}^{l_{2}}\right)^{+}, \omega} f_{g}\left(\chi_{2}^{l_{1}}, \chi_{4}^{l_{2}}\right)+\Im_{\rho, \eta,\left(\chi_{1}^{l_{1}}\right)^{+},\left(\chi_{4}^{l_{2}}\right)^{-}, \omega}^{\sigma} f_{g}\left(\chi_{2}^{l_{1}}, \chi_{3}^{l_{2}}\right)\right. \\
& \left.+\Im_{\rho, \eta,\left(\chi_{2}^{l_{1}}\right)^{-},\left(\chi_{3}^{l_{2}}\right)^{+}, \omega}^{\sigma} f_{g}\left(\chi_{1}^{l_{1}}, \chi_{4}^{l_{2}}\right)+\Im_{\rho, \eta,\left(\chi_{2}^{l_{1}}\right)^{-},\left(\chi_{4}^{l_{2}}\right)^{-}, \omega}^{\sigma} f_{g}\left(\chi_{1}^{l_{1}}, \chi_{3}^{l_{2}}\right)\right\} \\
& \leq \frac{\left(f\left(\chi_{1}, \chi_{3}\right)+f\left(\chi_{1}, \chi_{4}\right)+f\left(\chi_{2}, \chi_{3}\right)+f\left(\chi_{2}, \chi_{4}\right)\right)}{\mathfrak{F}_{\rho_{1}, \eta_{1}+1}^{\sigma_{1}}\left(\omega_{1}\left(\chi_{2}^{l_{1}}-\chi_{1}^{l_{1}}\right)^{\rho_{1}}\right) \mathfrak{F}_{\rho_{2}, \eta_{2}+1}^{\sigma_{2}}\left(\omega_{2}\left(\chi_{4}^{l_{2}}-\chi_{3}^{l_{2}}\right)^{\rho_{2}}\right)} \\
& \times \int_{0}^{1} \int_{0}^{1} \xi_{1}^{\eta_{1}-1} \xi_{2}^{\eta_{2}-1} \mathfrak{F}_{\rho_{1}, \eta_{1}}^{\sigma_{1}}\left[\omega_{1}\left(\chi_{2}^{l_{1}}-\chi_{1}^{l_{1}}\right)^{\rho_{1}} \xi_{1}^{\rho_{1}}\right] \mathfrak{F}_{\rho_{2}, \eta_{2}}^{\sigma_{2}}\left[\omega_{2}\left(\chi_{4}^{l_{2}}-\chi_{3}^{l_{2}}\right)^{\rho_{2}} \xi_{2}^{\rho_{2}}\right] \\
& \times\left(h_{1}\left(\xi_{1}\right)+h_{1}\left(1-\xi_{1}\right)\right)\left(h_{2}\left(\xi_{2}\right)+h_{2}\left(1-\xi_{2}\right)\right) d \xi_{2} d \xi_{1} .
\end{aligned}
$$

This rearranges to the proof of Theorem 3.1.

Remark 3.1 Theorem 3.1 with $l_{1}=l_{2}=1$ and $h_{1}\left(\xi_{1}\right)=h_{2}\left(\xi_{1}\right)=\xi_{1}$ becomes Theorem 2.1 in [39].

Remark 3.2 Theorem 3.1 with $l_{1}=l_{2}=1, \eta_{1}=\eta_{2}=\alpha, \sigma_{1}(0)=\sigma_{2}(0)=1, \omega_{1}=\omega_{2}=0$, and $h_{1}\left(\xi_{1}\right)=h_{2}\left(\xi_{1}\right)=\xi_{1}$ becomes Theorem 3 in [33].

Remark 3.3 Theorem 3.1 with $\eta_{1}=\eta_{2}=1, \sigma_{1}(0)=\sigma_{2}(0)=1$, and $\omega_{1}=\omega_{2}=0$ becomes Theorem 2.1 in [40].

Remark 3.4 Theorem 3.1 with $l_{1}=l_{2}=1, \eta_{1}=\eta_{2}=1, \sigma_{1}(0)=\sigma_{2}(0)=1, \omega_{1}=\omega_{2}=0$, and $h_{1}\left(\xi_{1}\right)=h_{2}\left(\xi_{1}\right)=h\left(\xi_{1}\right)$ becomes Theorem 7 in [35].

Theorem 3.2 Let $f: \Delta \rightarrow \mathbb{R}$ be an integrable and $\left(l_{1}, h_{1}\right)-\left(l_{2}, h_{2}\right)$-convex function on coordinates on $\Delta$. Then we have

$$
\begin{aligned}
& f\left(\left[\frac{\chi_{1}^{l_{1}}+\chi_{2}^{l_{1}}}{2}\right]^{\frac{1}{l_{1}}},\left[\frac{\chi_{3}^{l_{2}}+\chi_{4}^{l_{2}}}{2}\right]^{\frac{1}{l_{2}}}\right) \\
& \leq \frac{h_{1}\left(\frac{1}{2}\right)\left(\Im_{\rho_{1}, \eta_{1},\left(\chi_{1}^{l_{1}}\right)^{+}, \omega_{1}}^{\sigma_{g}}\left(\chi_{2}^{l_{1}}, \frac{\chi_{3}^{l_{2}}+\chi_{4}^{l_{2}}}{2}\right)+\Im_{\rho_{1}, \eta_{1},\left(\chi_{2}^{l_{1}}\right)^{\sigma_{1}, \omega_{1}}} f_{g}\left(\chi_{1}^{l_{1}}, \frac{\chi_{3}^{l_{2}}+\chi_{4}^{l_{2}}}{2}\right)\right)}{2\left(\chi_{2}^{l_{1}}-\chi_{1}^{l_{1}}\right)^{\eta_{1}} \mathfrak{F}_{\rho_{1}, \eta_{1}+1}^{\sigma_{1}}\left(\omega_{1}\left(\chi_{2}^{l_{1}}-\chi_{1}^{l_{1}}\right)^{\rho_{1}}\right)} \\
& \\
& \quad+\frac{h_{2}\left(\frac{1}{2}\right)\left(\Im_{\rho_{2}, \eta_{2},\left(\chi_{3}^{\sigma_{2}}\right)^{+}, \omega_{2}}^{\sigma_{2}} f_{g}\left(\frac{\chi_{1}^{l_{1}}+\chi_{2}^{l_{1}}}{2}, \chi_{4}^{l_{2}}\right)+\Im_{\rho_{2}, \eta_{2},\left(\chi_{4}^{l_{2}}\right)^{-}, \omega_{2}}^{\sigma_{2}} f_{g}\left(\frac{\chi_{1}^{l_{1}}+\chi_{2}^{l_{1}}}{2}, \chi_{3}^{l_{2}}\right)\right)}{2\left(\chi_{4}^{l_{2}}-\chi_{3}^{l_{2}}\right)^{\eta_{2}} \mathfrak{F}_{\rho_{2}, \eta_{2}+1}^{\sigma_{2}}\left(\omega_{2}\left(\chi_{4}^{l_{2}}-\chi_{3}^{l_{2}}\right)^{\rho_{2}}\right)} \\
& \leq h_{1}\left(\frac{1}{2}\right) \frac{f\left(\chi_{1},\left[\frac{\chi_{3}^{l_{2}}+\chi_{4}^{l_{2}}}{2}\right]^{\frac{1}{l_{2}}}\right)+f\left(\chi_{2},\left[\frac{\chi_{3}^{l_{2}}+\chi_{4}^{l_{2}}}{2}\right]^{\frac{1}{l_{2}}}\right)}{2 \mathfrak{F}_{\rho_{1}, \eta_{1}+1}^{\sigma_{1}}\left(\omega_{1}\left(\chi_{2}^{l_{1}}-\chi_{1}^{l_{1}}\right)^{\rho_{1}}\right)}
\end{aligned}
$$




$$
\begin{aligned}
& \times \int_{0}^{1} \xi_{1}^{\eta_{1}-1} \mathfrak{F}_{\rho_{1}, \eta_{1}}^{\sigma_{1}}\left[\omega_{1}\left(\chi_{2}^{l_{1}}-\chi_{1}^{l_{1}}\right)^{\rho_{1}} \xi_{1}^{\rho 1}\right]\left(h_{1}\left(\xi_{1}\right)+h_{1}\left(1-\xi_{1}\right)\right) d \xi_{1} \\
& +h_{2}\left(\frac{1}{2}\right) \frac{f\left(\left[\frac{\chi_{1}^{l_{1}}+\chi_{2}^{l_{1}}}{2}\right]^{\frac{1}{l_{1}}}, \chi_{3}\right)+f\left(\left[\frac{\chi_{1}^{l_{1}}+\chi_{2}^{l_{1}}}{2}\right]^{\frac{1}{l_{1}}}, \chi_{4}\right)}{2 \mathfrak{F}_{\rho_{2}, \eta_{2}+1}^{\sigma_{2}}\left(\omega_{2}\left(\chi_{4}^{l_{2}}-\chi_{3}^{l_{2}}\right)^{\rho_{2}}\right)} \\
& \times \int_{0}^{1} \xi_{2}^{\eta_{2}-1} \mathfrak{F}_{\rho_{2}, \eta_{2}}^{\sigma_{2}}\left[\omega_{2}\left(\chi_{4}^{l_{2}}-\chi_{3}^{l_{2}}\right)^{\rho_{2}} \xi_{2}^{\rho_{2}}\right]\left(h_{2}\left(\xi_{2}\right)+h_{2}\left(1-\xi_{2}\right)\right) d \xi_{2},
\end{aligned}
$$

where $f_{g}(x, y)=f\left(g_{1}(x), g_{2}(y)\right)$ with $g_{1}(x)=x^{\frac{1}{l_{1}}}$ and $g_{2}(y)=y^{\frac{1}{l_{2}}}$.

Proof Since $f$ is an $\left(l_{1}, h_{1}\right)-\left(l_{2}, h_{2}\right)$-convex function on coordinates on $\Delta$, the partial map$\operatorname{ping} f_{x}:\left[\chi_{3}, \chi_{4}\right] \rightarrow \mathbb{R}$ defined by $f_{x}(v)=f(x, v)$ is $\left(l_{2}, h_{2}\right)$-convex with respect to $v$ on $\left[\chi_{3}, \chi_{4}\right]$, and $f_{y}:\left[\chi_{1}, \chi_{2}\right] \rightarrow \mathbb{R}$ defined by $f_{y}(u)=f(u, y)$ is $\left(l_{1}, h_{1}\right)$-convex with respect to $u$ on $\left[\chi_{1}, \chi_{2}\right]$. So, we have

$$
\begin{aligned}
& f_{x}\left(\left[\frac{\chi_{3}^{l_{2}}+\chi_{4}^{l_{2}}}{2}\right]^{\frac{1}{l_{2}}}\right) \\
& \quad=f_{x}\left(\left[\frac{\left(\left(\xi_{2} \chi_{3}^{l_{2}}+\left(1-\xi_{2}\right) \chi_{4}^{l_{2}}\right)^{\frac{1}{l_{2}}}\right)^{l_{2}}+\left(\left(\left(1-\xi_{2}\right) \chi_{3}^{l_{2}}+\xi_{2} \chi_{4}^{l_{2}}\right)^{\frac{1}{l_{2}}}\right)^{l_{2}}}{2}\right]^{\frac{1}{l_{2}}}\right) \\
& \quad \leq h_{2}\left(\frac{1}{2}\right)\left(f_{x}\left(\left(\xi_{2} \chi_{3}^{l_{2}}+\left(1-\xi_{2}\right) \chi_{4}^{l_{2}}\right)^{\frac{1}{l_{2}}}\right)+f_{x}\left(\left(\left(1-\xi_{2}\right) \chi_{3}^{l_{2}}+\xi_{2} \chi_{4}^{l_{2}}\right)^{\frac{1}{l_{2}}}\right)\right) \\
& \quad \leq h_{2}\left(\frac{1}{2}\right)\left(h_{2}\left(\xi_{2}\right) f_{x}\left(\chi_{3}\right)+h_{2}\left(1-\xi_{2}\right) f_{x}\left(\chi_{4}\right)+h_{2}\left(1-\xi_{2}\right) f_{x}\left(\chi_{3}\right)+h_{2}\left(\xi_{2}\right) f_{x}\left(\chi_{4}\right)\right) \\
& \quad=h_{2}\left(\frac{1}{2}\right)\left(h_{2}\left(\xi_{2}\right)+h_{2}\left(1-\xi_{2}\right)\right)\left(f_{x}\left(\chi_{3}\right)+f_{x}\left(\chi_{4}\right)\right) .
\end{aligned}
$$

From (3.10), we get

$$
\begin{aligned}
\frac{1}{h_{2}\left(\frac{1}{2}\right)} f_{x}\left(\left[\frac{\chi_{3}^{l_{2}}+\chi_{4}^{l_{2}}}{2}\right]^{\frac{1}{l_{2}}}\right) & \leq f_{x}\left(\left(\xi_{2} \chi_{3}^{l_{2}}+\left(1-\xi_{2}\right) \chi_{4}^{l_{2}}\right)^{\frac{1}{l_{2}}}\right)+f_{x}\left(\left(\left(1-\xi_{2}\right) \chi_{3}^{l_{2}}+\xi_{2} \chi_{4}^{l_{2}}\right)^{\frac{1}{l_{2}}}\right) \\
& \leq\left(h_{2}\left(\xi_{2}\right)+h_{2}\left(1-\xi_{2}\right)\right)\left(f_{x}\left(\chi_{3}\right)+f_{x}\left(\chi_{4}\right)\right) .
\end{aligned}
$$

Multiplying (3.11) by $\xi_{2}^{\eta_{2}-1} \mathfrak{F}_{\rho_{2}, \eta_{2}}^{\sigma_{2}}\left[\omega_{2}\left(\chi_{4}^{l_{2}}-\chi_{3}^{l_{2}}\right)^{\rho_{2}} \xi_{2}^{\rho_{2}}\right]$ and then integrating the resulting inequalities with respect to $\xi_{2}$ on $[0,1]$, we obtain

$$
\begin{aligned}
& \frac{1}{h_{2}\left(\frac{1}{2}\right)} f_{x}\left(\left[\frac{\chi_{3}^{l_{2}}+\chi_{4}^{l_{2}}}{2}\right]^{\frac{1}{l_{2}}}\right) \int_{0}^{1} \xi_{2}^{\eta_{2}-1} \mathfrak{F}_{\rho_{2}, \eta_{2}}^{\sigma_{2}}\left[\omega_{2}\left(\chi_{4}^{l_{2}}-\chi_{3}^{l_{2}}\right)^{\rho_{2}} \xi_{2}^{\rho_{2}}\right] d \xi_{2} \\
& =\frac{1}{h_{2}\left(\frac{1}{2}\right)} f_{x}\left(\left[\frac{\chi_{3}^{l_{2}}+\chi_{4}^{l_{2}}}{2}\right]^{\frac{1}{l_{2}}}\right) \mathfrak{F}_{\rho_{2}, \eta_{2}+1}^{\sigma_{2}}\left(\omega_{2}\left(\chi_{4}^{l_{2}}-\chi_{3}^{l_{2}}\right)^{\rho_{2}}\right) \\
& \leq \int_{0}^{1} \xi_{2}^{\eta_{2}-1} \mathfrak{F}_{\rho_{2}, \eta_{2}}^{\sigma_{2}}\left[\omega_{2}\left(\chi_{4}^{l_{2}}-\chi_{3}^{l_{2}}\right)^{\rho_{2}} \xi_{2}^{\rho_{2}}\right] f_{x}\left(\left(\xi_{2} \chi_{3}^{l_{2}}+\left(1-\xi_{2}\right) \chi_{4}^{l_{2}}\right)^{\frac{1}{l_{2}}}\right) d \xi_{2} \\
& \quad+\int_{0}^{1} \xi_{2}^{\eta_{2}-1} \mathfrak{F}_{\rho_{2}, \eta_{2}}^{\sigma_{2}}\left[\omega_{2}\left(\chi_{4}^{l_{2}}-\chi_{3}^{l_{2}}\right)^{\rho_{2}} \xi_{2}^{\rho_{2}}\right] f_{x}\left(\left(\left(1-\xi_{2}\right) \chi_{3}^{l_{2}}+\xi_{2} \chi_{4}^{l_{2}}\right)^{\frac{1}{l_{2}}}\right) d \xi_{2} \\
& =\frac{1}{\left(\chi_{4}^{l_{2}}-\chi_{3}^{l_{2}}\right)^{\eta_{2}}} \int_{\chi_{3}^{l_{2}}}^{\chi_{4}^{l_{2}}}\left(\chi_{4}^{l_{2}}-w\right)^{\eta_{2}-1} \mathfrak{F}_{\rho_{2}, \eta_{2}}^{\sigma_{2}}\left[\omega_{2}\left(\chi_{4}^{l_{2}}-w\right)^{\rho_{2}}\right] f_{x}(w) d w
\end{aligned}
$$




$$
\begin{aligned}
& +\frac{1}{\left(\chi_{4}^{l_{2}}-\chi_{3}^{l_{2}}\right)^{\eta_{2}}} \int_{\chi_{3}^{l_{2}}}^{\chi_{4}^{l_{2}}}\left(w-\chi_{3}^{l_{2}}\right)^{\eta_{2}-1} \mathfrak{F}_{\rho_{2}, \eta_{2}}^{\sigma_{2}}\left[\omega_{2}\left(w-\chi_{3}^{l_{2}}\right)^{\rho_{2}}\right] f_{x}(w) d w \\
= & \frac{1}{\left(\chi_{4}^{l_{2}}-\chi_{3}^{l_{2}}\right)^{\eta_{2}}}\left(\Im_{\rho_{2}, \eta_{2},\left(\chi_{3}^{\sigma_{2}}\right)^{+}, \omega_{2}}^{\sigma_{2}} f_{x}\left(\chi_{4}^{l_{2}}\right)+\Im_{\rho_{2}, \eta_{2},\left(\chi_{4}^{\sigma_{2}}\right)^{\sigma_{2}}, \omega_{2}}^{\sigma_{2}} f_{x}\left(\chi_{3}^{l_{2}}\right)\right) \\
\leq & \left(f_{x}\left(\chi_{3}\right)+f_{x}\left(\chi_{4}\right)\right) \int_{0}^{1} \xi_{2}^{\eta_{2}-1} \mathfrak{F}_{\rho_{2}, \eta_{2}}^{\sigma_{2}}\left[\omega_{2}\left(\chi_{4}^{l_{2}}-\chi_{3}^{l_{2}}\right)^{\rho_{2}} \xi_{2}^{\rho_{2}}\right] \\
& \times\left(h_{2}\left(\xi_{2}\right)+h_{2}\left(1-\xi_{2}\right)\right) d \xi_{2} .
\end{aligned}
$$

This implies that

$$
\begin{aligned}
& \frac{1}{h_{2}\left(\frac{1}{2}\right)} f\left(x,\left[\frac{\chi_{3}^{l_{2}}+\chi_{4}^{l_{2}}}{2}\right]^{\frac{1}{l_{2}}}\right) \\
& \leq \frac{1}{\left(\chi_{4}^{l_{2}}-\chi_{3}^{l_{2}}\right)^{\eta_{2}} \mathfrak{F}_{\rho_{2}, \eta_{2}+1}^{\sigma_{2}}\left(\omega_{2}\left(\chi_{4}^{l_{2}}-\chi_{3}^{l_{2}}\right)^{\rho_{2}}\right)} \\
& \quad \times\left(\Im_{\rho_{2}, \eta_{2},\left(\chi_{3}^{\sigma_{2}}\right)^{+}, \omega_{2}}^{\sigma_{g}}\left(x^{l_{1}}, \chi_{4}^{l_{2}}\right)+\Im^{\sigma_{2}} \rho_{2, \eta_{2},\left(\chi_{4}^{l_{2}}\right)^{-}, \omega_{2}} f_{g}\left(x^{l_{1}}, \chi_{3}^{l_{2}}\right)\right) \\
& \leq \frac{f\left(x, \chi_{3}\right)+f\left(x, \chi_{4}\right)}{\mathfrak{F}_{\rho_{2}, \eta_{2}+1}^{\sigma_{2}}\left(\omega_{2}\left(\chi_{4}^{l_{2}}-\chi_{3}^{l_{2}}\right)^{\rho_{2}}\right)} \int_{0}^{1} \xi_{2}^{\eta_{2}-1} \mathfrak{F}_{\rho_{2}, \eta_{2}}^{\sigma_{2}}\left[\omega_{2}\left(\chi_{4}^{l_{2}}-\chi_{3}^{l_{2}}\right)^{\rho_{2}} \xi_{2}^{\rho_{2}}\right] \\
& \times\left(h_{2}\left(\xi_{2}\right)+h_{2}\left(1-\xi_{2}\right)\right) d \xi_{2} .
\end{aligned}
$$

Put $x=\left[\frac{\chi_{1}^{l_{1}}+\chi_{2}^{l_{1}}}{2}\right]^{\frac{1}{l_{1}}}$ into (3.13) to get

$$
\begin{aligned}
f( & {\left.\left[\frac{\chi_{1}^{l_{1}}+\chi_{2}^{l_{1}}}{2}\right]^{\frac{1}{l_{1}}},\left[\frac{\chi_{3}^{l_{2}}+\chi_{4}^{l_{2}}}{2}\right]^{\frac{1}{l_{2}}}\right) } \\
\leq & \frac{h_{2}\left(\frac{1}{2}\right)}{\left(\chi_{4}^{l_{2}}-\chi_{3}^{l_{2}}\right)^{\eta_{2}} \mathfrak{F}_{\rho_{2}, \eta_{2}+1}^{\sigma_{2}}\left(\omega_{2}\left(\chi_{4}^{l_{2}}-\chi_{3}^{l_{2}}\right)^{\rho_{2}}\right)} \\
& \times\left(\Im^{\sigma_{2}} \rho_{\rho_{2}, \eta_{2},\left(\chi_{3}^{l_{2}}\right)^{+}, \omega_{2}} f_{g}\left(\frac{\chi_{1}^{l_{1}}+\chi_{2}^{l_{1}}}{2}, \chi_{4}^{l_{2}}\right)+\Im_{\rho_{2}, \eta_{2},\left(\chi_{4}^{l_{2}}\right)^{-,}, \omega_{2}}^{\sigma_{2}} f_{g}\left(\frac{\chi_{1}^{l_{1}}+\chi_{2}^{l_{1}}}{2}, \chi_{3}^{l_{2}}\right)\right) \\
\leq & h_{2}\left(\frac{1}{2}\right) \frac{f\left(\left[\frac{\chi_{1}^{l_{1}}+\chi_{2}^{l_{1}}}{2}\right]^{\frac{1}{l_{1}}}, \chi_{3}\right)+f\left(\left[\frac{\chi_{1}^{l_{1}}+\chi_{2}^{l_{1}}}{2}\right]^{\frac{1}{l_{1}}}, \chi_{4}\right)}{\mathfrak{F}_{\rho_{2}, \eta_{2}+1}^{\sigma_{2}}\left(\omega_{2}\left(\chi_{4}^{l_{2}}-\chi_{3}^{l_{2}}\right)^{\rho_{2}}\right)} \\
& \times \int_{0}^{1} \xi_{2}^{\eta_{2}-1} \mathfrak{F}_{\rho_{2}, \eta_{2}}^{\sigma_{2}}\left[\omega_{2}\left(\chi_{4}^{l_{2}}-\chi_{3}^{l_{2}}\right)^{\rho_{2}} \xi_{2}^{\rho_{2}}\right]\left(h_{2}\left(\xi_{2}\right)+h_{2}\left(1-\xi_{2}\right)\right) d \xi_{2} .
\end{aligned}
$$

Similarly, we can deduce

$$
\begin{aligned}
f( & {\left.\left[\frac{\chi_{1}^{l_{1}}+\chi_{2}^{l_{1}}}{2}\right]^{\frac{1}{l_{1}}},\left[\frac{\chi_{3}^{l_{2}}+\chi_{4}^{l_{2}}}{2}\right]^{\frac{1}{l_{2}}}\right) } \\
\leq & \frac{h_{1}\left(\frac{1}{2}\right)}{\left(\chi_{2}^{l_{1}}-\chi_{1}^{l_{1}}\right)^{\eta_{1}} \mathfrak{F}_{\rho_{1}, \eta_{1}+1}^{\sigma_{1}}\left(\omega_{1}\left(\chi_{2}^{l_{1}}-\chi_{1}^{l_{1}}\right)^{\rho_{1}}\right)} \\
& \quad \times\left(\Im_{\rho_{1}, \eta_{1},\left(\chi_{1}^{\sigma_{1}}\right)^{+}, \omega_{1}}^{\sigma_{1}} f_{g}\left(\chi_{2}^{l_{1}}, \frac{\chi_{3}^{l_{2}}+\chi_{4}^{l_{2}}}{2}\right)+\Im_{\rho_{1}, \eta_{1},\left(\chi_{2}^{l_{1}}\right)^{-,}, \omega_{1}}^{\sigma_{1}} f_{g}\left(\chi_{1}^{l_{1}}, \frac{\chi_{3}^{l_{2}}+\chi_{4}^{l_{2}}}{2}\right)\right)
\end{aligned}
$$




$$
\begin{aligned}
\leq & h_{1}\left(\frac{1}{2}\right) \frac{f\left(\chi_{1},\left[\frac{\chi_{3}^{l_{2}}+\chi_{4}^{l_{2}}}{2}\right]^{\frac{1}{l_{2}}}\right)+f\left(\chi_{2},\left[\frac{\chi_{3}^{l_{2}}+\chi_{4}^{l_{2}}}{2}\right]^{\frac{1}{l_{2}}}\right)}{\mathfrak{F}_{\rho_{1}, \eta_{1}+1}^{\sigma_{1}}\left(\omega_{1}\left(\chi_{2}^{l_{1}}-\chi_{1}^{l_{1}}\right)^{\rho_{1}}\right)} \\
& \times \int_{0}^{1} \xi_{1}^{\eta_{1}-1} \mathfrak{F}_{\rho_{1}, \eta_{1}}^{\sigma_{1}}\left[\omega_{1}\left(\chi_{2}^{l_{1}}-\chi_{1}^{l_{1}}\right)^{\rho_{1}} \xi_{1}^{\rho 1}\right]\left(h_{1}\left(\xi_{1}\right)+h_{1}\left(1-\xi_{1}\right)\right) d \xi_{1} .
\end{aligned}
$$

By adding (3.14) and (3.15) together, and then multiplying the result by $\frac{1}{2}$, we get the desired result. Thus we get the proof of Theorem 3.2.

Remark 3.5 Theorem 3.2 with $l_{1}=l_{2}=1$ and $h_{1}\left(\xi_{1}\right)=h_{2}\left(\xi_{1}\right)=\xi_{1}$ becomes Theorem 2.2 in [39].

Lemma 3.1 Let $f: \Delta \rightarrow \mathbb{R}$ be a partial differentiable function on $\Delta$. If $\frac{\partial^{2} f}{\partial \xi_{1} \partial \xi_{2}} \in L(\Delta)$, then we have

$$
\begin{aligned}
& \frac{f\left(\chi_{1}, \chi_{3}\right)}{4 \chi_{1}^{1-l_{1}} \chi_{3}^{1-l_{2}}}+\frac{f\left(\chi_{1}, \chi_{4}\right)}{4 \chi_{1}^{1-l_{1}} \chi_{4}^{1-l_{2}}}+\frac{f\left(\chi_{2}, \chi_{3}\right)}{4 \chi_{2}^{1-l_{1}} \chi_{3}^{1-l_{2}}}+\frac{f\left(\chi_{2}, \chi_{4}\right)}{4 \chi_{2}^{1-l_{1}} \chi_{4}^{1-l_{2}}}-A \\
& +\frac{1}{4\left(\chi_{2}^{l_{1}}-\chi_{1}^{l_{1}}\right)^{\eta_{1}}\left(\chi_{4}^{l_{2}}-\chi_{3}^{l_{2}}\right)^{\eta_{2}} \mathfrak{F}_{\rho_{1}, \eta_{1}+1}^{\sigma_{1}}\left[\omega_{1}\left(\chi_{2}^{l_{1}}-\chi_{1}^{l_{1}}\right)^{\rho_{1}}\right] \mathfrak{F}_{\rho_{2}, \eta_{2}+1}^{\sigma_{2}}\left[\omega_{2}\left(\chi_{4}^{l_{2}}-\chi_{3}^{l_{2}}\right)^{\rho_{2}}\right]} \\
& \times\left(\Im_{\rho, \eta,\left(\chi_{2}^{l_{1}}\right)^{-},\left(\chi_{3}^{l_{2}}\right)^{+}, \omega} \chi_{1}^{l_{1}-1} \chi_{4}^{l_{2}-1} f_{g}\left(\chi_{1}^{l_{1}}, \chi_{4}^{l_{2}}\right)+\Im_{\rho, \eta,\left(\chi_{2}^{l_{1}}\right)^{-},\left(\chi_{4}^{l_{2}}\right)^{-}, \omega}^{\sigma} \chi_{1}^{l_{1}-1} \chi_{3}^{l_{2}-1} f_{g}\left(\chi_{1}^{l_{1}}, \chi_{3}^{l_{2}}\right)\right. \\
& \left.+\Im_{\rho, \eta,\left(\chi_{1}^{l_{1}}\right)^{+},\left(\chi_{4}^{l_{2}}\right)^{-}, \omega} \chi_{2}^{l_{1}-1} \chi_{3}^{l_{2}-1} f_{g}\left(\chi_{2}^{l_{1}}, \chi_{3}^{l_{2}}\right)+\Im_{\rho, \eta,\left(\chi_{1}^{l_{1}}\right)^{+},\left(\chi_{3}^{l_{2}}\right)^{+}, \omega}^{\sigma} \chi_{2}^{l_{1}-1} \chi_{4}^{l_{2}-1} f_{g}\left(\chi_{2}^{l_{1}}, \chi_{4}^{l_{2}}\right)\right) \\
& =\frac{\left(\chi_{2}^{l_{1}}-\chi_{1}^{l_{1}}\right)\left(\chi_{4}^{l_{2}}-\chi_{3}^{l_{2}}\right)}{4 l_{1} l_{2} \mathfrak{F}_{\rho_{1}, \eta_{1}+1}^{\sigma_{1}}\left[\omega_{1}\left(\chi_{2}^{l_{1}}-\chi_{1}^{l_{1}}\right)^{\rho_{1}}\right] \mathfrak{F}_{\rho_{2}, \eta_{2}+1}^{\sigma_{2}}\left[\omega_{2}\left(\chi_{4}^{l_{2}}-\chi_{3}^{l_{2}}\right)^{\rho_{2}}\right]} \\
& \times\left(\int_{0}^{1} \int_{0}^{1} \mathcal{B}\left(\xi_{1}, \xi_{2}\right) \frac{\partial^{2} f}{\partial \xi_{1} \partial \xi_{2}}\left(\left(\xi_{1} \chi_{1}^{l_{1}}+\left(1-\xi_{1}\right) \chi_{2}^{l_{1}}\right)^{\frac{1}{l_{1}}},\left(\xi_{2} \chi_{3}^{l_{2}}+\left(1-\xi_{2}\right) \chi_{4}^{l_{2}}\right)^{\frac{1}{l_{2}}}\right) d \xi_{1} d \xi_{2}\right. \\
& +\int_{0}^{1} \int_{0}^{1} \mathcal{B}\left(\xi_{1}, \xi_{2}\right) \frac{\partial^{2} f}{\partial \xi_{1} \partial \xi_{2}}\left(\left(\xi_{1} \chi_{1}^{l_{1}}+\left(1-\xi_{1}\right) \chi_{2}^{l_{1}}\right)^{\frac{1}{l_{1}}},\left(\left(1-\xi_{2}\right) \chi_{3}^{l_{2}}+\xi_{2} \chi_{4}^{l_{2}}\right)^{\frac{1}{l_{2}}}\right) d \xi_{1} d \xi_{2} \\
& +\int_{0}^{1} \int_{0}^{1} \mathcal{B}\left(\xi_{1}, \xi_{2}\right) \frac{\partial^{2} f}{\partial \xi_{1} \partial \xi_{2}}\left(\left(\left(1-\xi_{1}\right) \chi_{1}^{l_{1}}+\xi_{1} \chi_{2}^{l_{1}}\right)^{\frac{1}{l_{1}}},\left(\xi_{2} \chi_{3}^{l_{2}}+\left(1-\xi_{2}\right) \chi_{4}^{l_{2}}\right)^{\frac{1}{l_{2}}}\right) d \xi_{1} d \xi_{2} \\
& \left.+\int_{0}^{1} \int_{0}^{1} \mathcal{B}\left(\xi_{1}, \xi_{2}\right) \frac{\partial^{2} f}{\partial \xi_{1} \partial \xi_{2}}\left(\left(\left(1-\xi_{1}\right) \chi_{1}^{l_{1}}+\xi_{1} \chi_{2}^{l_{1}}\right)^{\frac{1}{l_{1}}},\left(\left(1-\xi_{2}\right) \chi_{3}^{l_{2}}+\xi_{2} \chi_{4}^{l_{2}}\right)^{\frac{1}{l_{2}}}\right) d \xi_{1} d \xi_{2}\right),
\end{aligned}
$$

where

$$
\mathcal{B}\left(\xi_{1}, \xi_{2}\right)=\xi_{1}^{\eta_{1}} \xi_{2}^{\eta_{2}} \mathfrak{F}_{\rho_{1}, \eta_{1}+1}^{\sigma_{1}}\left[\omega_{1}\left(\chi_{2}^{l_{1}}-\chi_{1}^{l_{1}}\right)^{\rho_{1}} \xi_{1}^{\rho_{1}}\right] \mathfrak{F}_{\rho_{2}, \eta_{2}+1}^{\sigma_{2}}\left[\omega_{2}\left(\chi_{4}^{l_{2}}-\chi_{3}^{l_{2}}\right)^{\rho_{2}} \xi_{2}^{\rho_{2}}\right]
$$

and

$$
\begin{aligned}
A= & \frac{1}{4\left(\chi_{4}^{l_{2}}-\chi_{3}^{l_{2}}\right)^{\eta_{2}} \mathfrak{F}_{\rho_{2}, \eta_{2}+1}^{\sigma_{2}}\left[\omega_{2}\left(\chi_{4}^{l_{2}}-\chi_{3}^{l_{2}}\right)^{\rho_{2}}\right]}\left(\frac{\Im_{\rho_{2}, \eta_{2},\left(\chi_{4}^{l_{2}}\right)^{-}, \omega_{2}}^{\sigma_{2}} \chi_{3}^{l_{2}-1} f_{g}\left(\chi_{1}^{l_{1}}, \chi_{3}^{l_{2}}\right)}{\chi_{1}^{1-l_{1}}}\right. \\
& +\frac{\Im_{\rho_{2}, \eta_{2},\left(\chi_{3}^{\sigma_{2}}\right)^{+}, \omega_{2}}^{l_{2}} \chi_{4}^{l_{2}-1} f_{g}\left(\chi_{1}^{l_{1}}, \chi_{4}^{l_{2}}\right)}{\chi_{1}^{1-l_{1}}}+\frac{\Im_{\rho_{2}, \eta_{2},\left(\chi_{4}^{\sigma_{2}}\right)^{-}, \omega_{2}}^{\sigma_{2}} \chi_{3}^{l_{2}-1} f_{g}\left(\chi_{2}^{l_{1}}, \chi_{3}^{l_{2}}\right)}{\chi_{2}^{1-l_{1}}}
\end{aligned}
$$




$$
\begin{aligned}
& \left.+\frac{\Im^{\rho_{2}, \eta_{2},\left(\chi_{3}^{l_{2}}\right)^{+}, \omega_{2}} \chi_{4}^{l_{2}-1} f_{g}\left(\chi_{2}^{l_{1}}, \chi_{4}^{l_{2}}\right)}{\chi_{2}^{1-l_{1}}}\right)+\frac{1}{4\left(\chi_{2}^{l_{1}}-\chi_{1}^{l_{1}}\right)^{\eta_{1}} \mathfrak{F}_{\rho_{1}, \eta_{1}+1}^{\sigma_{1}}\left[\omega_{1}\left(\chi_{2}^{l_{1}}-\chi_{1}^{l_{1}}\right)^{\rho_{1}}\right]} \\
& \times\left(\frac{\Im^{\rho_{1}, \eta_{1},\left(\chi_{2}^{l_{1}}\right)^{-}, \omega_{1}} \chi_{1}^{l_{1}-1} f_{g}\left(\chi_{1}^{l_{1}}, \chi_{3}^{l_{2}}\right)}{\chi_{3}^{1-l_{2}}}+\frac{\Im_{\rho_{1}, \eta_{1},\left(\chi_{2}^{l_{1}}\right)^{-}, \omega_{1}}^{\sigma_{1}} \chi_{1}^{l_{1}-1} f_{g}\left(\chi_{1}^{l_{1}}, \chi_{4}^{l_{2}}\right)}{\chi_{4}^{1-l_{2}}}\right. \\
& \left.+\frac{\Im^{\rho_{1}}{ }_{\rho_{1}, \eta_{1},\left(\chi_{1}^{l_{1}}\right)^{+}, \omega_{1}}^{l_{2}} \chi_{2}^{l_{1}-1} f_{g}\left(\chi_{2}^{l_{1}}, \chi_{3}^{l_{2}}\right)}{\chi_{3}^{l_{2}-1}}+\frac{\Im_{\rho_{1}, \eta_{1},\left(\chi_{1}^{l_{1}}\right)^{+}, \omega_{1}}^{\sigma_{1}} \chi_{2}^{l_{1}-1} f_{g}\left(\chi_{2}^{l_{1}}, \chi_{4}^{l_{2}}\right)}{\chi_{4}^{1-l_{2}}}\right),
\end{aligned}
$$

and $f_{g}(x, y)=f\left(g_{1}(x), g_{2}(y)\right)$ with $g_{1}(x)=x^{\frac{1}{l_{1}}}$ and $g_{2}(y)=y^{\frac{1}{l_{2}}}$.

\section{Proof Set}

$$
\hbar:=\hbar_{1}-\hbar_{2}-\hbar_{3}+\hbar_{4}
$$

where

$$
\begin{aligned}
\hbar_{1}:= & \int_{0}^{1} \int_{0}^{1} \xi_{1}^{\eta_{1}} \xi_{2}^{\eta_{2}} \mathfrak{F}_{\rho_{1}, \eta_{1}+1}^{\sigma_{1}}\left[\omega_{1}\left(\chi_{2}^{l_{1}}-\chi_{1}^{l_{1}}\right)^{\rho_{1}} \xi_{1}^{\rho_{1}}\right] \mathfrak{F}_{\rho_{2}, \eta_{2}+1}^{\sigma_{2}}\left[\omega_{2}\left(\chi_{4}^{l_{2}}-\chi_{3}^{l_{2}}\right)^{\rho_{2}} \xi_{2}^{\rho_{2}}\right] \\
& \times \frac{\partial^{2} f}{\partial \xi_{1} \partial \xi_{2}}\left(\left(\xi_{1} \chi_{1}^{l_{1}}+\left(1-\xi_{1}\right) \chi_{2}^{l_{1}}\right)^{\frac{1}{l_{1}}},\left(\xi_{2} \chi_{3}^{l_{2}}+\left(1-\xi_{2}\right) \chi_{4}^{l_{2}}\right)^{\frac{1}{l_{2}}}\right) d \xi_{1} d \xi_{2} ; \\
\hbar_{2}:= & \int_{0}^{1} \int_{0}^{1} \xi_{1}^{\eta_{1}} \xi_{2}^{\eta_{2}} \mathfrak{F}_{\rho_{1}, \eta_{1}+1}^{\sigma_{1}}\left[\omega_{1}\left(\chi_{2}^{l_{1}}-\chi_{1}^{l_{1}}\right)^{\rho_{1}} \xi_{1}^{\rho_{1}}\right] \mathfrak{F}_{\rho_{2}, \eta_{2}+1}^{\sigma_{2}}\left[\omega_{2}\left(\chi_{4}^{l_{2}}-\chi_{3}^{l_{2}}\right)^{\rho_{2}} \xi_{2}^{\rho_{2}}\right] \\
& \times \frac{\partial^{2} f}{\partial \xi_{1} \partial \xi_{2}}\left(\left(\xi_{1} \chi_{1}^{l_{1}}+\left(1-\xi_{1}\right) \chi_{2}^{l_{1}}\right)^{\frac{1}{l_{1}}},\left(\left(1-\xi_{2}\right) \chi_{3}^{l_{2}}+\xi_{2} \chi_{4}^{l_{2}}\right)^{\frac{1}{l_{2}}}\right) d \xi_{1} d \xi_{2} ; \\
\hbar_{3}:= & \int_{0}^{1} \int_{0}^{1} \xi_{1}^{\eta_{1}} \xi_{2}^{\eta_{2}} \mathfrak{F}_{\rho_{1}, \eta_{1}+1}^{\sigma_{1}}\left[\omega_{1}\left(\chi_{2}^{l_{1}}-\chi_{1}^{l_{1}}\right)^{\rho_{1}} \xi_{1}^{\rho_{1}}\right] \mathfrak{F}_{\rho_{2}, \eta_{2}+1}^{\sigma_{2}}\left[\omega_{2}\left(\chi_{4}^{l_{2}}-\chi_{3}^{l_{2}}\right)^{\rho_{2}} \xi_{2}^{\rho_{2}}\right] \\
& \times \frac{\partial^{2} f}{\partial \xi_{1} \partial \xi_{2}}\left(\left(\left(1-\xi_{1}\right) \chi_{1}^{l_{1}}+\xi_{1} \chi_{2}^{l_{1}}\right)^{\frac{1}{l_{1}}},\left(\xi_{2} \chi_{3}^{l_{2}}+\left(1-\xi_{2}\right) \chi_{4}^{l_{2}}\right)^{\frac{1}{l_{2}}}\right) d \xi_{1} d \xi_{2} ; \\
\hbar_{4}:= & \int_{0}^{1} \int_{0}^{1} \xi_{1}^{\eta_{1}} \xi_{2}^{\eta_{2}} \mathfrak{F}_{\rho_{1}, \eta_{1}+1}^{\sigma_{1}}\left[\omega_{1}\left(\chi_{2}^{l_{1}}-\chi_{1}^{l_{1}}\right)^{\rho_{1}} \xi_{1}^{\rho_{1}}\right] \mathfrak{F}_{\rho_{2}, \eta_{2}+1}^{\sigma_{2}}\left[\omega_{2}\left(\chi_{4}^{l_{2}}-\chi_{3}^{l_{2}}\right)^{\rho_{2}} \xi_{2}^{\rho_{2}}\right] \\
& \times \frac{\partial^{2} f}{\partial \xi_{1} \partial \xi_{2}}\left(\left(\left(1-\xi_{1}\right) \chi_{1}^{l_{1}}+\xi_{1} \chi_{2}^{l_{1}}\right)^{\frac{1}{l_{1}}},\left(\left(1-\xi_{2}\right) \chi_{3}^{l_{2}}+\xi_{2} \chi_{4}^{l_{2}}\right)^{\frac{1}{l_{2}}}\right) d \xi_{1} d \xi_{2} .
\end{aligned}
$$

Integrating by parts $\hbar_{1}$, we have

$$
\begin{aligned}
\hbar_{1}= & \int_{0}^{1} \xi_{2}^{\eta_{2}} \mathfrak{F}_{\rho_{2}, \eta_{2}+1}^{\sigma_{2}}\left[\omega_{2}\left(\chi_{4}^{l_{2}}-\chi_{3}^{l_{2}}\right)^{\rho_{2}} \xi_{2}^{\rho_{2}}\right]\left(\int_{0}^{1} \xi_{1}^{\eta_{1}} \mathfrak{F}_{\rho_{1}, \eta_{1}+1}^{\sigma_{1}}\left[\omega_{1}\left(\chi_{2}^{l_{1}}-\chi_{1}^{l_{1}}\right)^{\rho_{1}} \xi_{1}^{\rho_{1}}\right]\right. \\
& \left.\times \frac{\partial^{2} f}{\partial \xi_{1} \partial \xi_{2}}\left(\left(\xi_{1} \chi_{1}^{l_{1}}+\left(1-\xi_{1}\right) \chi_{2}^{l_{1}}\right)^{\frac{1}{l_{1}}},\left(\xi_{2} \chi_{3}^{l_{2}}+\left(1-\xi_{2}\right) \chi_{4}^{l_{2}}\right)^{\frac{1}{l_{2}}}\right) d \xi_{1}\right) d \xi_{2} \\
= & \frac{l_{1} \mathfrak{F}_{\rho_{1}, \eta_{1}+1}^{\sigma_{1}}\left[\omega_{1}\left(\chi_{2}^{l_{1}}-\chi_{1}^{l_{1}}\right)^{\rho_{1}}\right]}{\left(\chi_{1}^{l_{1}}-\chi_{2}^{l_{1}}\right) \chi_{1}^{1-l_{1}}} \int_{0}^{1} \xi_{2}^{\eta_{2}} \mathfrak{F}_{\rho_{2}, \eta_{2}+1}^{\sigma_{2}}\left[\omega_{2}\left(\chi_{4}^{l_{2}}-\chi_{3}^{l_{2}}\right)^{\rho_{2}} \xi_{2}^{\rho_{2}}\right] \\
& \times \frac{\partial f}{\partial \xi_{2}}\left(\chi_{1},\left(\xi_{2} \chi_{3}^{l_{2}}+\left(1-\xi_{2}\right) \chi_{4}^{l_{2}}\right)^{\frac{1}{l_{2}}}\right) d \xi_{2}
\end{aligned}
$$




$$
\begin{aligned}
& -\int_{0}^{1} \frac{l_{1} \xi_{1}^{\eta_{1}-1} \mathfrak{F}_{\rho_{1}, \eta_{1}}^{\sigma_{1}}\left[\omega_{1}\left(\chi_{2}^{l_{1}}-\chi_{1}^{l_{1}}\right)^{\rho_{1}} \xi_{1}^{\rho_{1}}\right]}{\left(\chi_{1}^{l_{1}}-\chi_{2}^{l_{1}}\right)\left(\xi_{1} \chi_{1}^{l_{1}}+\left(1-\xi_{1}\right) \chi_{2}^{l_{1}}\right)^{\frac{1}{l_{1}}-1}}\left(\int_{0}^{1} \xi_{2}^{\eta_{2}} \mathfrak{F}_{\rho_{2}, \eta_{2}+1}^{\sigma_{2}}\left[\omega_{2}\left(\chi_{4}^{l_{2}}-\chi_{3}^{l_{2}}\right)^{\rho_{2}} \xi_{2}^{\rho_{2}}\right]\right. \\
& \left.\times \frac{\partial f}{\partial \xi_{2}}\left(\left(\xi_{1} \chi_{1}^{l_{1}}+\left(1-\xi_{1}\right) \chi_{2}^{l_{1}}\right)^{\frac{1}{l_{1}}},\left(\xi_{2} \chi_{3}^{l_{2}}+\left(1-\xi_{2}\right) \chi_{4}^{l_{2}}\right)^{\frac{1}{l_{2}}}\right) d \xi_{2}\right) d \xi_{1} \\
& =\frac{l_{1} \mathfrak{F}_{\rho_{1}, \eta_{1}+1}^{\sigma_{1}}\left[\omega_{1}\left(\chi_{2}^{l_{1}}-\chi_{1}^{l_{1}}\right)^{\rho_{1}}\right]}{\left(\chi_{2}^{l_{1}}-\chi_{1}^{l_{1}}\right)} \frac{l_{2} \mathfrak{F}_{\rho_{2}, \eta_{2}+1}^{\sigma_{2}}\left[\omega_{2}\left(\chi_{4}^{l_{2}}-\chi_{3}^{l_{2}}\right)^{\rho_{2}}\right]}{\left(\chi_{4}^{l_{2}}-\chi_{3}^{l_{2}}\right)} \frac{f\left(\chi_{1}, \chi_{3}\right)}{\chi_{1}^{1-l_{1}} \chi_{3}^{1-l_{2}}} \\
& -\frac{l_{1} l_{2} \mathfrak{F}_{\rho_{1}, \eta_{1}+1}^{\sigma_{1}}\left[\omega_{1}\left(\chi_{2}^{l_{1}}-\chi_{1}^{l_{1}}\right)^{\rho_{1}}\right]}{\left(\chi_{2}^{l_{1}}-\chi_{1}^{l_{1}}\right)\left(\chi_{4}^{l_{2}}-\chi_{3}^{l_{2}}\right) \chi_{1}^{1-l_{1}}} \int_{0}^{1} \xi_{2}^{\eta_{2}-1} \mathfrak{F}_{\rho_{2}, \eta_{2}}^{\sigma_{2}}\left[\omega_{2}\left(\chi_{4}^{l_{2}}-\chi_{3}^{l_{2}}\right)^{\rho_{2}} \xi_{2}^{\rho_{2}}\right] \\
& \times\left(\xi_{2} \chi_{3}^{l_{2}}+\left(1-\xi_{2}\right) \chi_{4}^{l_{2}}\right)^{1-\frac{1}{l_{2}}} f\left(\chi_{1},\left(\xi_{2} \chi_{3}^{l_{2}}+\left(1-\xi_{2}\right) \chi_{4}^{l_{2}}\right)^{\frac{1}{l_{2}}}\right) d \xi_{2} \\
& -\frac{l_{1} l_{2} \mathfrak{F}_{\rho_{2}, \eta_{2}+1}^{\sigma_{2}}\left[\omega_{2}\left(\chi_{4}^{l_{2}}-\chi_{3}^{l_{2}}\right)^{\rho_{2}}\right]}{\left(\chi_{2}^{l_{1}}-\chi_{1}^{l_{1}}\right)\left(\chi_{4}^{l_{2}}-\chi_{3}^{l_{2}}\right) \chi_{3}^{1-l_{2}}} \int_{0}^{1} \xi_{1}^{\eta_{1}-1} \mathfrak{F}_{\rho_{1}, \eta_{1}}^{\sigma_{1}}\left[\omega_{1}\left(\chi_{2}^{l_{1}}-\chi_{1}^{l_{1}}\right)^{\rho_{1}} \xi_{1}^{\rho_{1}}\right] \\
& \times\left(\xi_{1} \chi_{1}^{l_{1}}+\left(1-\xi_{1}\right) \chi_{2}^{l_{1}}\right)^{1-\frac{1}{l_{1}}} f\left(\left(\xi_{1} \chi_{1}^{l_{1}}+\left(1-\xi_{1}\right) \chi_{2}^{l_{1}}\right)^{\frac{1}{l_{1}}}, \chi_{3}\right) d \xi_{1} \\
& +\frac{l_{1} l_{2}}{\left(\chi_{2}^{l_{1}}-\chi_{1}^{l_{1}}\right)\left(\chi_{4}^{l_{2}}-\chi_{3}^{l_{2}}\right)} \int_{0}^{1} \int_{0}^{1} \xi_{1}^{\eta_{1}-1} \mathfrak{F}_{\rho_{1}, \eta_{1}}^{\sigma_{1}}\left[\omega_{1}\left(\chi_{2}^{l_{1}}-\chi_{1}^{l_{1}}\right)^{\rho_{1}} \xi_{1}^{\rho_{1}}\right] \\
& \times \xi_{2}^{\eta_{2}-1} \mathfrak{F}_{\rho_{2}, \eta_{2}}^{\sigma_{2}}\left[\omega_{2}\left(\chi_{4}^{l_{2}}-\chi_{3}^{l_{2}}\right)^{\rho_{2}} \xi_{2}^{\rho_{2}}\right] \\
& \times\left(\xi_{1} \chi_{1}^{l_{1}}+\left(1-\xi_{1}\right) \chi_{2}^{l_{1}}\right)^{1-\frac{1}{l_{1}}}\left(\xi_{2} \chi_{3}^{l_{2}}+\left(1-\xi_{2}\right) \chi_{4}^{l_{2}}\right)^{1-\frac{1}{l_{2}}} \\
& \times f\left(\left(\xi_{1} \chi_{1}^{l_{1}}+\left(1-\xi_{1}\right) \chi_{2}^{l_{1}}\right)^{\frac{1}{l_{1}}},\left(\xi_{2} \chi_{3}^{l_{2}}+\left(1-\xi_{2}\right) \chi_{4}^{l_{2}}\right)^{\frac{1}{l_{2}}}\right) d \xi_{2} d \xi_{1} .
\end{aligned}
$$

By the change of variables, we get

$$
\begin{aligned}
\hbar_{1}= & \frac{l_{1} \mathfrak{F}_{\rho_{1}, \eta_{1}+1}^{\sigma_{1}}\left[\omega_{1}\left(\chi_{2}^{l_{1}}-\chi_{1}^{l_{1}}\right)^{\rho_{1}}\right]}{\left(\chi_{2}^{l_{1}}-\chi_{1}^{l_{1}}\right)} \frac{l_{2} \mathfrak{F}_{\rho_{2}, \eta_{2}+1}^{\sigma_{2}}\left[\omega_{2}\left(\chi_{4}^{l_{2}}-\chi_{3}^{l_{2}}\right)^{\rho_{2}}\right]}{\left(\chi_{4}^{l_{2}}-\chi_{3}^{l_{2}}\right)} \frac{f\left(\chi_{1}, \chi_{3}\right)}{\chi_{1}^{1-l_{1}} \chi_{3}^{1-l_{2}}} \\
& -\frac{l_{1} l_{2} \mathfrak{F}_{\rho_{1}, \eta_{1}+1}^{\sigma_{1}}\left[\omega_{1}\left(\chi_{2}^{l_{1}}-\chi_{1}^{l_{1}}\right)^{\rho_{1}}\right]}{\left(\chi_{2}^{l_{1}}-\chi_{1}^{l_{1}}\right)\left(\chi_{4}^{l_{2}}-\chi_{3}^{l_{2}}\right)^{\eta_{2}+1} \chi_{1}^{1-l_{1}}} \\
& \times \int_{\chi_{3}^{l_{2}}}^{\chi_{4}^{l_{2}}}\left(\chi_{4}^{l_{2}}-y\right)^{\eta_{2}-1} \mathfrak{F}_{\rho_{2}, \eta_{2}}^{\sigma_{2}}\left[\omega_{2}\left(\chi_{4}^{l_{2}}-y\right)^{\rho_{2}}\right] y^{1-\frac{1}{l_{2}}} f_{g}\left(\chi_{1}^{l_{1}}, y\right) d y \\
& -\frac{l_{1} l_{2} \mathfrak{F}_{\rho_{2}, \eta_{2}+1}^{\sigma_{2}}\left[\omega_{2}\left(\chi_{4}^{l_{2}}-\chi_{3}^{l_{2}}\right)^{\rho_{2}}\right]}{\left(\chi_{2}^{l_{1}}-\chi_{1}^{l_{1}}\right)^{\eta_{1}+1}\left(\chi_{4}^{l_{2}}-\chi_{3}^{l_{2}}\right) \chi_{3}^{1-l_{2}}} \\
& \times \int_{\chi_{1}^{l_{1}}}^{\chi_{2}^{l_{1}}}\left(\chi_{2}^{l_{1}}-x\right)^{\eta_{1}-1} \mathfrak{F}_{\rho_{1}, \eta_{1}}^{\sigma_{1}}\left[\omega_{1}\left(\chi_{2}^{l_{1}}-x\right)^{\rho_{1}}\right] x^{1-\frac{1}{l_{1}}} f_{g}\left(x, \chi_{3}^{l_{2}}\right) d x \\
& +\frac{l_{1} l_{2}}{\left(\chi_{2}^{l_{1}}-\chi_{1}^{l_{1}}\right)^{\eta_{1}+1}\left(\chi_{4}^{l_{2}}-\chi_{3}^{l_{2}}\right)^{\eta_{2}+1}} \int_{\chi_{1}^{l_{1}}}^{\chi_{1}^{l_{1}}} \int_{\chi_{3}^{l_{2}}}^{\chi_{4}^{l_{2}}}\left(\chi_{2}^{l_{1}}-x\right)^{\eta_{1}-1} \mathfrak{F}_{\rho_{1}, \eta_{1}}^{\sigma_{1}}\left[\omega_{1}\left(\chi_{2}^{l_{1}}-x\right)^{\rho_{1}}\right] \\
& \times\left(\chi_{4}^{l_{2}}-y\right)^{\eta_{2}-1} \mathfrak{F}_{\rho_{2}, \eta_{2}}^{\sigma_{2}}\left[\omega_{2}\left(\chi_{4}^{l_{2}}-y\right)^{\rho_{2}}\right] x^{1-\frac{1}{l_{1}}} y^{1-\frac{1}{l_{2}}} f_{g}(x, y) d y d x .
\end{aligned}
$$

Making use of Definition 2.3 in (3.19), we get

$$
\hbar_{1}=\frac{l_{1} l_{2} \mathfrak{F}_{\rho_{1}, \eta_{1}+1}^{\sigma_{1}}\left[\omega_{1}\left(\chi_{2}^{l_{1}}-\chi_{1}^{l_{1}}\right)^{\rho_{1}}\right] \mathfrak{F}_{\rho_{2}, \eta_{2}+1}^{\sigma_{2}}\left[\omega_{2}\left(\chi_{4}^{l_{2}}-\chi_{3}^{l_{2}}\right)^{\rho_{2}}\right]}{\left(\chi_{2}^{l_{1}}-\chi_{1}^{l_{1}}\right)\left(\chi_{4}^{l_{2}}-\chi_{3}^{l_{2}}\right)} \frac{f\left(\chi_{1}, \chi_{3}\right)}{\chi_{1}^{1-l_{1}} \chi_{3}^{1-l_{2}}}
$$




$$
\begin{aligned}
& -\frac{l_{1} l_{2} \mathfrak{F}_{\rho_{1}, \eta_{1}+1}^{\sigma_{1}}\left[\omega_{1}\left(\chi_{2}^{l_{1}}-\chi_{1}^{l_{1}}\right)^{\rho_{1}}\right]}{\left(\chi_{2}^{l_{1}}-\chi_{1}^{l_{1}}\right)\left(\chi_{4}^{l_{2}}-\chi_{3}^{l_{2}}\right)^{\eta_{2}+1}} \chi_{1}^{l_{1}-1} \Im_{\rho_{2}, \eta_{2},\left(\chi_{3}^{l_{2}}\right)^{+}, \omega_{2}}^{\sigma_{2}} \chi_{4}^{l_{2}-1} f_{g}\left(\chi_{1}^{l_{1}}, \chi_{4}^{l_{2}}\right) \\
& -\frac{l_{1} l_{2} \mathfrak{F}_{\rho_{2}, \eta_{2}+1}^{\sigma_{2}}\left[\omega_{2}\left(\chi_{4}^{l_{2}}-\chi_{3}^{l_{2}}\right)^{\rho_{2}}\right]}{\left(\chi_{2}^{l_{1}}-\chi_{1}^{l_{1}}\right)^{\eta_{1}+1}\left(\chi_{4}^{l_{2}}-\chi_{3}^{l_{2}}\right)} \chi_{3}^{l_{2}-1} \Im_{\rho_{1}, \eta_{1},\left(\chi_{1}^{l_{1}}\right)^{+}, \omega_{1}}^{\sigma_{1}} \chi_{2}^{l_{1}-1} f_{g}\left(\chi_{2}^{l_{1}}, \chi_{3}^{l_{2}}\right) \\
& +\frac{l_{1} l_{2}}{\left(\chi_{2}^{l_{1}}-\chi_{1}^{l_{1}}\right)^{\eta_{1}+1}\left(\chi_{4}^{l_{2}}-\chi_{3}^{l_{2}}\right)^{\eta_{2}+1}} \Im_{\rho, \eta,\left(\chi_{1}^{\sigma}\right)^{+},\left(\chi_{3}^{l_{2}}\right)^{+}, \omega}^{l_{1}} \chi_{2}^{l_{1}-1} \chi_{4}^{l_{2}-1} f_{g}\left(\chi_{2}^{l_{1}}, \chi_{4}^{l_{2}}\right) .
\end{aligned}
$$

Likewise, we can deduce

$$
\begin{aligned}
& \hbar_{2}=-\frac{l_{1} l_{2} \mathfrak{F}_{\rho_{1}, \eta_{1}+1}^{\sigma_{1}}\left[\omega_{1}\left(\chi_{2}^{l_{1}}-\chi_{1}^{l_{1}}\right)^{\rho_{1}}\right] \mathfrak{F}_{\rho_{2}, \eta_{2}+1}^{\sigma_{2}}\left[\omega_{2}\left(\chi_{4}^{l_{2}}-\chi_{3}^{l_{2}}\right)^{\rho_{2}}\right]}{\left(\chi_{2}^{l_{1}}-\chi_{1}^{l_{1}}\right)\left(\chi_{4}^{l_{2}}-\chi_{3}^{l_{2}}\right)} \frac{f\left(\chi_{1}, \chi_{4}\right)}{\chi_{1}^{1-l_{1}} \chi_{4}^{1-l_{2}}}
\end{aligned}
$$

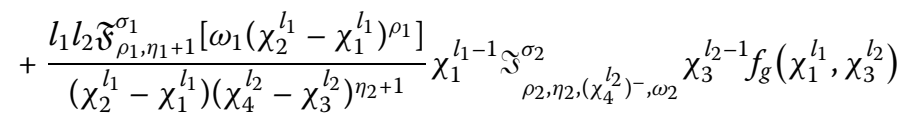

$$
\begin{aligned}
& +\frac{l_{1} l_{2} \widetilde{F}_{\rho_{2}, \eta_{2}+1}^{\sigma_{2}}\left[\omega_{2}\left(\chi_{4}^{l_{2}}-\chi_{3}^{l_{2}}\right)^{\rho_{2}}\right]}{\left(\chi_{2}^{l_{1}}-\chi_{1}^{l_{1}}\right)^{\eta_{1}+1}\left(\chi_{4}^{l_{2}}-\chi_{3}^{l_{2}}\right)} \chi_{4}^{l_{2}-1} \Im_{\rho_{1}, \eta_{1}, a\left(\chi_{1}^{\sigma_{1}}\right)^{+}, \omega_{1}}^{\sigma_{1}} \chi_{2}^{l_{1}-1} f_{g}\left(\chi_{2}^{l_{1}}, \chi_{4}^{l_{2}}\right) \\
& -\frac{l_{1} l_{2}}{\left(\chi_{2}^{l_{1}}-\chi_{1}^{l_{1}}\right)^{\eta_{1}+1}\left(\chi_{4}^{l_{2}}-\chi_{3}^{l_{2}}\right)^{\eta_{2}+1}} \Im_{\rho, \eta,\left(\chi_{1}^{l_{1}}\right)^{+},\left(\chi_{4}^{l_{2}}\right)^{-}, \omega} \chi_{2}^{l_{1}-1} \chi_{3}^{l_{2}-1} f_{g}\left(\chi_{2}^{l_{1}}, \chi_{3}^{l_{2}}\right), \\
& \hbar_{3}=-\frac{l_{1} \mathfrak{F}_{\rho_{1}, \eta_{1}+1}^{\sigma_{1}}\left[\omega_{1}\left(\chi_{2}^{l_{1}}-\chi_{1}^{l_{1}}\right)^{\rho_{1}}\right]}{\left(\chi_{2}^{l_{1}}-\chi_{1}^{l_{1}}\right)} \frac{l_{2} \mathfrak{F}_{\rho_{2}, \eta_{2}+1}^{\sigma_{2}}\left[\omega_{2}\left(\chi_{4}^{l_{2}}-\chi_{3}^{l_{2}}\right)^{\rho_{2}}\right]}{\left(\chi_{4}^{l_{2}}-\chi_{3}^{l_{2}}\right)} \frac{f\left(\chi_{2}, \chi_{3}\right)}{\chi_{2}^{1-l_{1}} \chi_{3}^{1-l_{2}}}
\end{aligned}
$$

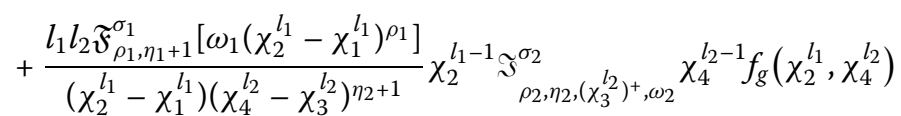

$$
\begin{aligned}
& +\frac{l_{1} l_{2} \mathfrak{F}_{\rho_{2}, \eta_{2}+1}^{\sigma_{2}}\left[\omega_{2}\left(\chi_{4}^{l_{2}}-\chi_{3}^{l_{2}}\right)^{\rho_{2}}\right]}{\left(\chi_{2}^{l_{1}}-\chi_{1}^{l_{1}}\right)^{\eta_{1}+1}\left(\chi_{4}^{l_{2}}-\chi_{3}^{l_{2}}\right)} \chi_{3}^{l_{2}-1} \mathfrak{\Im}_{\rho_{1}, \eta_{1},\left(\chi_{2}^{\sigma_{1}}\right)^{l_{1}, \omega_{1}}} \chi_{1}^{l_{1}-1} f_{g}\left(\chi_{1}^{l_{1}}, \chi_{3}^{l_{2}}\right) \\
& -\frac{l_{1} l_{2}}{\left(\chi_{2}^{l_{1}}-\chi_{1}^{l_{1}}\right)^{\eta_{1}+1}\left(\chi_{4}^{l_{2}}-\chi_{3}^{l_{2}}\right)^{\eta_{2}+1}} \Im_{\rho, \eta,\left(\chi_{2}^{l_{1}}\right)^{-},\left(\chi_{3}^{l_{2}}\right)^{+}, \omega}^{\chi_{1}^{l_{1}-1}} \chi_{4}^{l_{2}-1} f_{g}\left(\chi_{1}^{l_{1}}, \chi_{4}^{l_{2}}\right),
\end{aligned}
$$

and finally

$$
\begin{aligned}
\hbar_{4}= & \frac{l_{1} \mathfrak{F}_{\rho_{1}, \eta_{1}+1}^{\sigma_{1}}\left[\omega_{1}\left(\chi_{2}^{l_{1}}-\chi_{1}^{l_{1}}\right)^{\rho_{1}}\right]}{\left(\chi_{2}^{l_{1}}-\chi_{1}^{l_{1}}\right)} \frac{l_{2} \mathfrak{F}_{\rho_{2}, \eta_{2}+1}^{\sigma_{2}}\left[\omega_{2}\left(\chi_{4}^{l_{2}}-\chi_{3}^{l_{2}}\right)^{\rho_{2}}\right]}{\left(\chi_{4}^{l_{2}}-\chi_{3}^{l_{2}}\right)} \frac{f\left(\chi_{2}, \chi_{4}\right)}{\chi_{2}^{1-l_{1}} \chi_{4}^{1-l_{2}}} \\
& -\frac{l_{1} l_{2} \mathfrak{F}_{\rho_{1}, \eta_{1}+1}^{\sigma_{1}}\left[\omega_{1}\left(\chi_{2}^{l_{1}}-\chi_{1}^{l_{1}}\right)^{\rho_{1}}\right]}{\left(\chi_{2}^{l_{1}}-\chi_{1}^{l_{1}}\right)\left(\chi_{4}^{l_{2}}-\chi_{3}^{l_{2}}\right)^{\eta_{2}+1}} \chi_{2}^{l_{1}-1} \Im_{\Im_{2}}^{\sigma_{2}}{ }_{\rho_{2}, \eta_{2},\left(\chi_{4}^{l_{2}}\right)^{-}, \omega_{2}}^{l_{2}} \chi_{3}^{l_{2}-1} f_{g}\left(\chi_{2}^{l_{1}}, \chi_{3}^{l_{2}}\right) \\
& -\frac{l_{1} l_{2} \mathfrak{F}_{\rho_{2}, \eta_{2}+1}^{\sigma_{2}}\left[\omega_{2}\left(\chi_{4}^{l_{2}}-\chi_{3}^{l_{2}}\right)^{\rho_{2}}\right]}{\left(\chi_{2}^{l_{1}}-\chi_{1}^{l_{1}}\right)^{\eta_{1}+1}\left(\chi_{4}^{l_{2}}-\chi_{3}^{l_{2}}\right)} \chi_{4}^{l_{2}-1} \Im_{\Im_{1}, \eta_{1},\left(\chi_{2}^{l_{1}}\right)^{-}, \omega_{1}}^{\sigma_{1}} \chi_{1}^{l_{1}-1} f_{g}\left(\chi_{1}^{l_{1}}, \chi_{4}^{l_{2}}\right) \\
& +\frac{l_{1} l_{2}}{\left(\chi_{2}^{l_{1}}-\chi_{1}^{l_{1}}\right)^{\eta_{1}+1}\left(\chi_{4}^{l_{2}}-\chi_{3}^{l_{2}}\right)^{\eta_{2}+1}} \Im_{\rho, \eta,\left(\chi_{2}^{l_{1}}\right)^{-},\left(\chi_{4}^{l_{2}}\right)^{-}, \omega}^{\sigma} \chi_{1}^{l_{1}-1} \chi_{3}^{l_{2}-1} f_{g}\left(\chi_{1}^{l_{1}}, \chi_{3}^{l_{2}}\right) .
\end{aligned}
$$

Making use of (3.20)-(3.23) in (3.18) and then multiplying by

$$
\frac{\left(\chi_{2}^{l_{1}}-\chi_{1}^{l_{1}}\right)\left(\chi_{4}^{l_{2}}-\chi_{3}^{l_{2}}\right)}{4 l_{1} l_{2} \mathfrak{F}_{\rho_{1}, \eta_{1}+1}^{\sigma_{1}}\left[\omega_{1}\left(\chi_{2}^{l_{1}}-\chi_{1}^{l_{1}}\right)^{\rho_{1}}\right] \mathfrak{F}_{\rho_{2}, \eta_{2}+1}^{\sigma_{2}}\left[\omega_{2}\left(\chi_{4}^{l_{2}}-\chi_{3}^{l_{2}}\right)^{\rho_{2}}\right]},
$$

we arrive at the desired result. Thus we get the proof of Lemma 3.1. 
Theorem 3.3 Let $f: \Delta \rightarrow \mathbb{R}$ be a partial differentiable function on $\Delta$. If $\left|\frac{\partial^{2} f}{\partial \xi_{1} \partial \xi_{2}}\right|$ is an $\left(l_{1}, h_{1}\right)-\left(l_{2}, h_{2}\right)$-convex function on coordinates on $\Delta$, then we have

$$
\begin{aligned}
& \mid \frac{f\left(\chi_{1}, \chi_{3}\right)}{4 \chi_{1}^{1-l_{1}} \chi_{3}^{1-l_{2}}}+\frac{f\left(\chi_{1}, \chi_{4}\right)}{4 \chi_{1}^{1-l_{1}} \chi_{4}^{1-l_{2}}}+\frac{f\left(\chi_{2}, \chi_{3}\right)}{4 \chi_{2}^{1-l_{1}} \chi_{3}^{1-l_{2}}}+\frac{f\left(\chi_{2}, \chi_{4}\right)}{4 \chi_{2}^{1-l_{1}} \chi_{4}^{1-l_{2}}}-A \\
& +\frac{1}{4\left(\chi_{2}^{l_{1}}-\chi_{1}^{l_{1}}\right)^{\eta_{1}}\left(\chi_{4}^{l_{2}}-\chi_{3}^{l_{2}}\right)^{\eta_{2}} \mathfrak{F}_{\rho_{1}, \eta_{1}+1}^{\sigma_{1}}\left[\omega_{1}\left(\chi_{2}^{l_{1}}-\chi_{1}^{l_{1}}\right)^{\rho_{1}}\right] \mathfrak{F}_{\rho_{2}, \eta_{2}+1}^{\sigma_{2}}\left[\omega_{2}\left(\chi_{4}^{l_{2}}-\chi_{3}^{l_{2}}\right)^{\rho_{2}}\right]} \\
& \times\left(\Im_{\rho, \eta,\left(\chi_{2}^{l_{1}}\right)^{-},\left(\chi_{3}^{l_{2}}\right)^{+}, \omega}\left(\frac{f_{g}\left(\chi_{1}^{l_{1}}, \chi_{4}^{l_{2}}\right)}{\chi_{1}^{1-l_{1}} \chi_{4}^{1-l_{2}}}\right)+\Im_{\rho, \eta,\left(\chi_{2}^{l_{1}}\right)^{-},\left(\chi_{4}^{l_{2}}\right)^{-,}, \omega}\left(\frac{f_{g}\left(\chi_{1}^{l_{1}}, \chi_{3}^{l_{2}}\right)}{\chi_{1}^{1-l_{1}} \chi_{3}^{1-l_{2}}}\right)\right. \\
& \left.+\Im_{\rho, \eta,\left(\chi_{1}^{l_{1}}\right)^{+},\left(\chi_{4}^{l_{2}}\right)^{-}, \omega}^{\sigma}\left(\frac{f_{g}\left(\chi_{2}^{l_{1}}, \chi_{3}^{l_{2}}\right)}{\chi_{2}^{1-l_{1}} \chi_{3}^{1-l_{2}}}\right)+\Im_{\rho, \eta,\left(\chi_{1}^{l_{1}}\right)^{+},\left(\chi_{3}^{l_{2}}\right)^{+}, \omega}^{\sigma}\left(\frac{f_{g}\left(\chi_{2}^{l_{1}}, \chi_{4}^{l_{2}}\right)}{\chi_{2}^{1-l_{1}} \chi_{4}^{1-l_{2}}}\right)\right) \mid \\
& \leq \frac{\left(\chi_{2}^{l_{1}}-\chi_{1}^{l_{1}}\right)\left(\chi_{4}^{l_{2}}-\chi_{3}^{l_{2}}\right)}{4 l_{1} l_{2} \mathfrak{F}_{\rho_{1}, \eta_{1}+1}^{\sigma_{1}}\left[\omega_{1}\left(\chi_{2}^{l_{1}}-\chi_{1}^{l_{1}}\right)^{\rho_{1}}\right] \mathfrak{F}_{\rho_{2}, \eta_{2}+1}^{\sigma_{2}}\left[\omega_{2}\left(\chi_{4}^{l_{2}}-\chi_{3}^{l_{2}}\right)^{\rho_{2}}\right]} \\
& \times\left(\int_{0}^{1} \int_{0}^{1} \mathcal{B}\left(\xi_{1}, \xi_{2}\right)\left(h_{1}\left(1-\xi_{1}\right)+h_{1}\left(\xi_{1}\right)\right)\left(h_{2}\left(1-\xi_{2}\right)+h_{2}\left(\xi_{2}\right)\right) d \xi_{1} d \xi_{2}\right) \\
& \times\left(\left|\frac{\partial^{2} f}{\partial \xi_{1} \partial \xi_{2}}\left(\chi_{1}, \chi_{3}\right)\right|+\left|\frac{\partial^{2} f}{\partial \xi_{1} \partial \xi_{2}}\left(\chi_{1}, \chi_{4}\right)\right|+\left|\frac{\partial^{2} f}{\partial \xi_{1} \partial \xi_{2}}\left(\chi_{2}, \chi_{3}\right)\right|+\left|\frac{\partial^{2} f}{\partial \xi_{1} \partial \xi_{2}}\left(\chi_{2}, \chi_{4}\right)\right|\right),
\end{aligned}
$$

where $\mathcal{B}\left(\xi_{1}, \xi_{2}\right)$ and $A$ are as in (3.16) and (3.17), respectively, and $f_{g}(x, y)=f\left(g_{1}(x), g_{2}(y)\right)$ with $g_{1}(x)=x^{\frac{1}{l_{1}}}$ and $g_{2}(y)=y^{\frac{1}{l_{2}}}$.

Proof By Lemma 3.1 and the properties of modulus, we have

$$
\begin{aligned}
& \mid \frac{f\left(\chi_{1}, \chi_{3}\right)}{4 \chi_{1}^{1-l_{1}} \chi_{3}^{1-l_{2}}}+\frac{f\left(\chi_{1}, \chi_{4}\right)}{4 \chi_{1}^{1-l_{1}} \chi_{4}^{1-l_{2}}}+\frac{f\left(\chi_{2}, \chi_{3}\right)}{4 \chi_{2}^{1-l_{1}} \chi_{3}^{1-l_{2}}}+\frac{f\left(\chi_{2}, \chi_{4}\right)}{4 \chi_{2}^{1-l_{1}} \chi_{4}^{1-l_{2}}}-A \\
& +\frac{1}{4\left(\chi_{2}^{l_{1}}-\chi_{1}^{l_{1}}\right)^{\eta_{1}}\left(\chi_{4}^{l_{2}}-\chi_{3}^{l_{2}}\right)^{\eta_{2}} \mathfrak{F}_{\rho_{1}, \eta_{1}+1}^{\sigma_{1}}\left[\omega_{1}\left(\chi_{2}^{l_{1}}-\chi_{1}^{l_{1}}\right)^{\rho_{1}}\right] \mathfrak{F}_{\rho_{2}, \eta_{2}+1}^{\sigma_{2}}\left[\omega_{2}\left(\chi_{4}^{l_{2}}-\chi_{3}^{l_{2}}\right)^{\rho_{2}}\right]} \\
& \times\left(\Im_{\rho, \eta,\left(\chi_{2}^{l_{1}}\right)^{-},\left(\chi_{3}^{l_{2}}\right)^{+}, \omega}\left(\frac{f_{g}\left(\chi_{1}^{l_{1}}, \chi_{4}^{l_{2}}\right)}{\chi_{1}^{1-l_{1}} \chi_{4}^{1-l_{2}}}\right)+\Im_{\rho, \eta,\left(\chi_{2}^{l_{1}}\right)^{-},\left(\chi_{4}^{l_{2}}\right)^{-}, \omega}^{\sigma}\left(\frac{f_{g}\left(\chi_{1}^{l_{1}}, \chi_{3}^{l_{2}}\right)}{\chi_{1}^{1-l_{1}} \chi_{3}^{1-l_{2}}}\right)\right. \\
& \left.+\Im_{\rho, \eta,\left(\chi_{1}^{l_{1}}\right)^{+},\left(\chi_{4}^{l_{2}}\right)^{-}, \omega}^{\sigma}\left(\frac{f_{g}\left(\chi_{2}^{l_{1}}, \chi_{3}^{l_{2}}\right)}{\chi_{2}^{1-l_{1}} \chi_{3}^{1-l_{2}}}\right)+\Im_{\rho, \eta,\left(\chi_{1}^{l_{1}}\right)^{+},\left(\chi_{3}^{l_{2}}\right)^{+}, \omega}^{\sigma}\left(\frac{f_{g}\left(\chi_{2}^{l_{1}}, \chi_{4}^{l_{2}}\right)}{\chi_{2}^{1-l_{1}} \chi_{4}^{1-l_{2}}}\right)\right) \mid \\
& \leq \frac{\left(\chi_{2}^{l_{1}}-\chi_{1}^{l_{1}}\right)\left(\chi_{4}^{l_{2}}-\chi_{3}^{l_{2}}\right)}{4 l_{1} l_{2} \mathfrak{F}_{\rho_{1}, \eta_{1}+1}^{\sigma_{1}}\left[\omega_{1}\left(\chi_{2}^{l_{1}}-\chi_{1}^{l_{1}}\right)^{\rho_{1}}\right] \mathfrak{F}_{\rho_{2}, \eta_{2}+1}^{\sigma_{2}}\left[\omega_{2}\left(\chi_{4}^{l_{2}}-\chi_{3}^{l_{2}}\right)^{\rho_{2}}\right]} \\
& \times\left(\int_{0}^{1} \int_{0}^{1} \mathcal{B}\left(\xi_{1}, \xi_{2}\right) \mid \frac{\partial^{2} f}{\partial \xi_{1} \partial \xi_{2}}\left(\left(\xi_{1} \chi_{1}^{l_{1}}+\left(1-\xi_{1}\right) \chi_{2}^{l_{1}}\right)^{\frac{1}{l_{1}}},\right.\right. \\
& \left.\left(\xi_{2} \chi_{3}^{l_{2}}+\left(1-\xi_{2}\right) \chi_{4}^{l_{2}}\right)^{\frac{1}{l_{2}}}\right) \mid d \xi_{1} d \xi_{2} \\
& +\int_{0}^{1} \int_{0}^{1} \mathcal{B}\left(\xi_{1}, \xi_{2}\right) \mid \frac{\partial^{2} f}{\partial \xi_{1} \partial \xi_{2}}\left(\left(\xi_{1} \chi_{1}^{l_{1}}+\left(1-\xi_{1}\right) \chi_{2}^{l_{1}}\right)^{\frac{1}{l_{1}}}\right. \\
& \left.\left(\left(1-\xi_{2}\right) \chi_{3}^{l_{2}}+\xi_{2} \chi_{4}^{l_{2}}\right)^{\frac{1}{l_{2}}}\right) \mid d \xi_{1} d \xi_{2}
\end{aligned}
$$




$$
\begin{aligned}
& +\int_{0}^{1} \int_{0}^{1} \mathcal{B}\left(\xi_{1}, \xi_{2}\right) \mid \frac{\partial^{2} f}{\partial \xi_{1} \partial \xi_{2}}\left(\left(\left(1-\xi_{1}\right) \chi_{1}^{l_{1}}+\xi_{1} \chi_{2}^{l_{1}}\right)^{\frac{1}{l_{1}}}\right. \\
& \left.\left(\xi_{2} \chi_{3}^{l_{2}}+\left(1-\xi_{2}\right) \chi_{4}^{l_{2}}\right)^{\frac{1}{l_{2}}}\right) \mid d \xi_{1} d \xi_{2} \\
& +\int_{0}^{1} \int_{0}^{1} \mathcal{B}\left(\xi_{1}, \xi_{2}\right) \mid \frac{\partial^{2} f}{\partial \xi_{1} \partial \xi_{2}}\left(\left(\left(1-\xi_{1}\right) \chi_{1}^{l_{1}}+\xi_{1} \chi_{2}^{l_{1}}\right)^{\frac{1}{l_{1}}}\right. \\
& \left.\left.\left(\left(1-\xi_{2}\right) \chi_{3}^{l_{2}}+\xi_{2} \chi_{4}^{l_{2}}\right)^{\frac{1}{l_{2}}}\right) \mid d \xi_{1} d \xi_{2}\right) .
\end{aligned}
$$

Using the $\left(l_{1}, h_{1}\right)-\left(l_{2}, h_{2}\right)$-convexity of $\left|\frac{\partial^{2} f}{\partial \xi_{1} \partial \xi_{2}}\right|$ on coordinates, we obtain

$$
\begin{aligned}
& \mid \frac{f\left(\chi_{1}, \chi_{3}\right)}{4 \chi_{1}^{1-l_{1}} \chi_{3}^{1-l_{2}}}+\frac{f\left(\chi_{1}, \chi_{4}\right)}{4 \chi_{1}^{1-l_{1}} \chi_{4}^{1-l_{2}}}+\frac{f\left(\chi_{2}, \chi_{3}\right)}{4 \chi_{2}^{1-l_{1}} \chi_{3}^{1-l_{2}}}+\frac{f\left(\chi_{2}, \chi_{4}\right)}{4 \chi_{2}^{1-l_{1}} \chi_{4}^{1-l_{2}}}-A \\
& +\frac{1}{4\left(\chi_{2}^{l_{1}}-\chi_{1}^{l_{1}}\right)^{\eta_{1}}\left(\chi_{4}^{l_{2}}-\chi_{3}^{l_{2}}\right)^{\eta_{2}} \mathfrak{F}_{\rho_{1}, \eta_{1}+1}^{\sigma_{1}}\left[\omega_{1}\left(\chi_{2}^{l_{1}}-\chi_{1}^{l_{1}}\right)^{\rho_{1}}\right] \mathfrak{F}_{\rho_{2}, \eta_{2}+1}^{\sigma_{2}}\left[\omega_{2}\left(\chi_{4}^{l_{2}}-\chi_{3}^{l_{2}}\right)^{\rho_{2}}\right]} \\
& \times\left(\Im_{\rho, \eta,\left(\chi_{2}^{l_{1}}\right)^{-},\left(\chi_{3}^{l_{2}}\right)^{+}, \omega}^{\sigma}\left(\frac{f_{g}\left(\chi_{1}^{l_{1}}, \chi_{4}^{l_{2}}\right)}{\chi_{1}^{1-l_{1}} \chi_{4}^{1-l_{2}}}\right)+\mathfrak{\Im}_{\rho, \eta,\left(\chi_{2}^{l_{1}}\right)^{-},\left(\chi_{4}^{l_{2}}\right)^{-}, \omega}^{\sigma}\left(\frac{f_{g}\left(\chi_{1}^{l_{1}}, \chi_{3}^{l_{2}}\right)}{\chi_{1}^{1-l_{1}} \chi_{3}^{1-l_{2}}}\right)\right. \\
& \left.+\Im_{\rho, \eta,\left(\chi_{1}^{l_{1}}\right)^{+},\left(\chi_{4}^{l_{2}}\right)^{-}, \omega}^{\sigma}\left(\frac{f_{g}\left(\chi_{2}^{l_{1}}, \chi_{3}^{l_{2}}\right)}{\chi_{2}^{1-l_{1}} \chi_{3}^{1-l_{2}}}\right)+\Im_{\rho, \eta,\left(\chi_{1}^{l_{1}}\right)^{+},\left(\chi_{3}^{l_{2}}\right)^{+}, \omega}^{\sigma}\left(\frac{f_{g}\left(\chi_{2}^{l_{1}}, \chi_{4}^{l_{2}}\right)}{\chi_{2}^{1-l_{1}} \chi_{4}^{1-l_{2}}}\right)\right) \mid \\
& \leq \frac{\left(\chi_{2}^{l_{1}}-\chi_{1}^{l_{1}}\right)\left(\chi_{4}^{l_{2}}-\chi_{3}^{l_{2}}\right)}{4 l_{1} l_{2} \mathfrak{F}_{\rho_{1}, \eta_{1}+1}^{\sigma_{1}}\left[\omega_{1}\left(\chi_{2}^{l_{1}}-\chi_{1}^{l_{1}}\right)^{\rho_{1}}\right] \mathfrak{F}_{\rho_{2}, \eta_{2}+1}^{\sigma_{2}}\left[\omega_{2}\left(\chi_{4}^{l_{2}}-\chi_{3}^{l_{2}}\right)^{\rho_{2}}\right]} \\
& \times\left(\int _ { 0 } ^ { 1 } \int _ { 0 } ^ { 1 } \mathcal { B } ( \xi _ { 1 } , \xi _ { 2 } ) \left(h_{1}\left(\xi_{1}\right) h_{2}\left(\xi_{2}\right)\left|\frac{\partial^{2} f}{\partial \xi_{1} \partial \xi_{2}}\left(\chi_{1}, \chi_{3}\right)\right|\right.\right. \\
& +h_{1}\left(\xi_{1}\right) h_{2}\left(1-\xi_{2}\right)\left|\frac{\partial^{2} f}{\partial \xi_{1} \partial \xi_{2}}\left(\chi_{1}, \chi_{4}\right)\right|+h_{1}\left(1-\xi_{1}\right) h_{2}\left(\xi_{2}\right)\left|\frac{\partial^{2} f}{\partial \xi_{1} \partial \xi_{2}}\left(\chi_{2}, \chi_{3}\right)\right| \\
& \left.+h_{1}\left(1-\xi_{1}\right) h_{2}\left(1-\xi_{2}\right)\left|\frac{\partial^{2} f}{\partial \xi_{1} \partial \xi_{2}}\left(\chi_{2}, \chi_{4}\right)\right|\right) d \xi_{1} d \xi_{2} \\
& +\int_{0}^{1} \int_{0}^{1} \mathcal{B}\left(\xi_{1}, \xi_{2}\right)\left(h_{1}\left(\xi_{1}\right) h_{2}\left(1-\xi_{2}\right)\left|\frac{\partial^{2} f}{\partial \xi_{1} \partial \xi_{2}}\left(\chi_{1}, \chi_{3}\right)\right|\right. \\
& +h_{1}\left(\xi_{1}\right) h_{2}\left(\xi_{2}\right)\left|\frac{\partial^{2} f}{\partial \xi_{1} \partial \xi_{2}}\left(\chi_{1}, \chi_{4}\right)\right|+h_{1}\left(1-\xi_{1}\right) h_{2}\left(1-\xi_{2}\right)\left|\frac{\partial^{2} f}{\partial \xi_{1} \partial \xi_{2}}\left(\chi_{2}, \chi_{3}\right)\right| \\
& \left.+h_{1}\left(1-\xi_{1}\right) h_{2}\left(\xi_{2}\right)\left|\frac{\partial^{2} f}{\partial \xi_{1} \partial \xi_{2}}\left(\chi_{2}, \chi_{4}\right)\right|\right) d \xi_{1} d \xi_{2} \\
& +\int_{0}^{1} \int_{0}^{1} \mathcal{B}\left(\xi_{1}, \xi_{2}\right)\left(h_{1}\left(1-\xi_{1}\right) h_{2}\left(\xi_{2}\right)\left|\frac{\partial^{2} f}{\partial \xi_{1} \partial \xi_{2}}\left(\chi_{1}, \chi_{3}\right)\right|\right. \\
& +h_{1}\left(1-\xi_{1}\right) h_{2}\left(1-\xi_{2}\right)\left|\frac{\partial^{2} f}{\partial \xi_{1} \partial \xi_{2}}\left(\chi_{1}, \chi_{4}\right)\right|+h_{1}\left(\xi_{1}\right) h_{2}\left(\xi_{2}\right)\left|\frac{\partial^{2} f}{\partial \xi_{1} \partial \xi_{2}}\left(\chi_{2}, \chi_{3}\right)\right| \\
& \left.+h_{1}\left(\xi_{1}\right) h_{2}\left(1-\xi_{2}\right)\left|\frac{\partial^{2} f}{\partial \xi_{1} \partial \xi_{2}}\left(\chi_{2}, \chi_{4}\right)\right|\right) d \xi_{1} d \xi_{2} \\
& +\int_{0}^{1} \int_{0}^{1} \mathcal{B}\left(\xi_{1}, \xi_{2}\right)\left(h_{1}\left(1-\xi_{1}\right) h_{2}\left(1-\xi_{2}\right)\left|\frac{\partial^{2} f}{\partial \xi_{1} \partial \xi_{2}}\left(\chi_{1}, \chi_{3}\right)\right|\right.
\end{aligned}
$$




$$
\begin{aligned}
& +h_{1}\left(1-\xi_{1}\right) h_{2}\left(\xi_{2}\right)\left|\frac{\partial^{2} f}{\partial \xi_{1} \partial \xi_{2}}\left(\chi_{1}, \chi_{4}\right)\right|+h_{1}\left(\xi_{1}\right) h_{2}\left(1-\xi_{2}\right)\left|\frac{\partial^{2} f}{\partial \xi_{1} \partial \xi_{2}}\left(\chi_{2}, \chi_{3}\right)\right| \\
& \left.\left.+h_{1}\left(\xi_{1}\right) h_{2}\left(\xi_{2}\right)\left|\frac{\partial^{2} f}{\partial \xi_{1} \partial \xi_{2}}\left(\chi_{2}, \chi_{4}\right)\right|\right) d \xi_{1} d \xi_{2}\right) \\
= & \frac{\left(\chi_{2}^{l_{1}}-\chi_{1}^{l_{1}}\right)\left(\chi_{4}^{l_{2}}-\chi_{3}^{l_{2}}\right)}{4 l_{1} l_{2} \mathfrak{F}_{\rho_{1}, \eta_{1}+1}^{\sigma_{1}}\left[\omega_{1}\left(\chi_{2}^{l_{1}}-\chi_{1}^{l_{1}}\right)^{\rho_{1}}\right] \mathfrak{F}_{\rho_{2}, \eta_{2}+1}^{\sigma_{2}}\left[\omega_{2}\left(\chi_{4}^{l_{2}}-\chi_{3}^{l_{2}}\right)^{\rho_{2}}\right]} \\
& \times\left(\int_{0}^{1} \int_{0}^{1} \mathcal{B}\left(\xi_{1}, \xi_{2}\right)\left(h_{1}\left(1-\xi_{1}\right)+h_{1}\left(\xi_{1}\right)\right)\left(\left(h_{2}\left(1-\xi_{2}\right)+h_{2}\left(\xi_{2}\right)\right)\right) d \xi_{1} d \xi_{2}\right) \\
& \times\left(\left|\frac{\partial^{2} f}{\partial \xi_{1} \partial \xi_{2}}\left(\chi_{1}, \chi_{3}\right)\right|+\left|\frac{\partial^{2} f}{\partial \xi_{1} \partial \xi_{2}}\left(\chi_{1}, \chi_{4}\right)\right|+\left|\frac{\partial^{2} f}{\partial \xi_{1} \partial \xi_{2}}\left(\chi_{2}, \chi_{3}\right)\right|+\left|\frac{\partial^{2} f}{\partial \xi_{1} \partial \xi_{2}}\left(\chi_{2}, \chi_{4}\right)\right|\right) .
\end{aligned}
$$

This completely ends the proof of Theorem 3.3.

Corollary 3.1 Theorem 3.3 with $\left|\frac{\partial^{2} f}{\partial \xi_{1} \partial \xi_{2}}\right| \leq K$ gives the new inequality:

$$
\begin{aligned}
& \mid \frac{f\left(\chi_{1}, \chi_{3}\right)}{4 \chi_{1}^{1-l_{1}} \chi_{3}^{1-l_{2}}}+\frac{f\left(\chi_{1}, \chi_{4}\right)}{4 \chi_{1}^{1-l_{1}} \chi_{4}^{1-l_{2}}}+\frac{f\left(\chi_{2}, \chi_{3}\right)}{4 \chi_{2}^{1-l_{1}} \chi_{3}^{1-l_{2}}}+\frac{f\left(\chi_{2}, \chi_{4}\right)}{4 \chi_{2}^{1-l_{1}} \chi_{4}^{1-l_{2}}}-A \\
& \quad+\frac{1}{4\left(\chi_{2}^{l_{1}}-\chi_{1}^{l_{1}}\right)^{\eta_{1}}\left(\chi_{4}^{l_{2}}-\chi_{3}^{l_{2}}\right)^{\eta_{2}} \mathfrak{F}_{\rho_{1}, \eta_{1}+1}^{\sigma_{1}}\left[\omega_{1}\left(\chi_{2}^{l_{1}}-\chi_{1}^{l_{1}}\right)^{\rho_{1}}\right] \mathfrak{F}_{\rho_{2}, \eta_{2}+1}^{\sigma_{2}}\left[\omega_{2}\left(\chi_{4}^{l_{2}}-\chi_{3}^{l_{2}}\right)^{\rho_{2}}\right]} \\
& \quad \times\left(\Im_{\rho, \eta,\left(\chi_{2}^{l_{1}}\right)^{-},\left(\chi_{3}^{l_{2}}\right)^{+}, \omega}\left(\frac{f_{g}\left(\chi_{1}^{l_{1}}, \chi_{4}^{l_{2}}\right)}{\chi_{1}^{1-l_{1}} \chi_{4}^{1-l_{2}}}\right)+\Im_{\rho, \eta,\left(\chi_{2}^{l_{1}}\right)^{-},\left(\chi_{4}^{l_{2}}\right)^{-}, \omega}\left(\frac{f_{g}\left(\chi_{1}^{l_{1}}, \chi_{3}^{l_{2}}\right)}{\chi_{1}^{1-l_{1}} \chi_{3}^{1-l_{2}}}\right)\right. \\
& \left.\quad+\Im_{\rho, \eta,\left(\chi_{1}^{l_{1}}\right)^{+},\left(\chi_{4}^{l_{2}}\right)^{-}, \omega}\left(\frac{f_{g}\left(\chi_{2}^{l_{1}}, \chi_{3}^{l_{2}}\right)}{\chi_{2}^{1-l_{1}} \chi_{3}^{1-l_{2}}}\right)+\Im_{\rho, \eta,}^{\sigma}\left(\chi_{1}^{\left.l_{1}\right)^{+},\left(\chi_{3}^{l_{2}}\right)^{+}, \omega}\left(\frac{f_{g}\left(\chi_{2}^{l_{1}}, \chi_{4}^{l_{2}}\right)}{\chi_{2}^{1-l_{1}} \chi_{4}^{1-l_{2}}}\right)\right)\right) \\
& \quad \frac{K\left(\chi_{2}^{l_{1}}-\chi_{1}^{l_{1}}\right)\left(\chi_{4}^{l_{2}}-\chi_{3}^{l_{2}}\right)}{l_{1} l_{2} \mathfrak{F}_{\rho_{1}, \eta_{1}+1}^{\sigma_{1}}\left[\omega_{1}\left(\chi_{2}^{l_{1}}-\chi_{1}^{l_{1}}\right)^{\rho_{1}}\right] \mathfrak{F}_{\rho_{2}, \eta_{2}+1}^{\sigma_{2}}\left[\omega_{2}\left(\chi_{4}^{l_{2}}-\chi_{3}^{l_{2}}\right)^{\rho_{2}}\right]} \\
& \quad \times\left(\int_{0}^{1} \int_{0}^{1} \mathcal{B}\left(\xi_{1}, \xi_{2}\right)\left(h_{1}\left(1-\xi_{1}\right)+h_{1}\left(\xi_{1}\right)\right)\left(h_{2}\left(1-\xi_{2}\right)+h_{2}\left(\xi_{2}\right)\right) d \xi_{1} d \xi_{2}\right) .
\end{aligned}
$$

Remark 3.6 Theorem 3.3 with $l_{1}=l_{2}=1$ and $h_{1}\left(\xi_{1}\right)=h_{2}\left(\xi_{1}\right)=\xi_{1}$ becomes Theorem 3.2 in [39].

Remark 3.7 Theorem 3.3 with $l_{1}=l_{2}=1, \eta_{1}=\eta_{2}=\alpha, \sigma_{1}(0)=\sigma_{2}(0)=1, \omega_{1}=\omega_{2}=0$, and $h_{1}\left(\xi_{1}\right)=h_{2}\left(\xi_{1}\right)=\xi_{1}$ becomes Theorem 3 in [33].

Theorem 3.4 Let $f: \Delta \rightarrow \mathbb{R}$ be a partial differentiable function on $\Delta$. If $\left|\frac{\partial^{2} f}{\partial \xi_{1} \partial \xi_{2}}\right|^{q}$ is an $\left(l_{1}, h_{1}\right)-\left(l_{2}, h_{2}\right)$-convex function on coordinates on $\Delta$, then for $q>1$ and $\frac{1}{p}+\frac{1}{q}=1$, we have

$$
\begin{aligned}
& \mid \frac{f\left(\chi_{1}, \chi_{3}\right)}{4 \chi_{1}^{1-l_{1}} \chi_{3}^{1-l_{2}}}+\frac{f\left(\chi_{1}, \chi_{4}\right)}{4 \chi_{1}^{1-l_{1}} \chi_{4}^{1-l_{2}}}+\frac{f\left(\chi_{2}, \chi_{3}\right)}{4 \chi_{2}^{1-l_{1}} \chi_{3}^{1-l_{2}}}+\frac{f\left(\chi_{2}, \chi_{4}\right)}{4 \chi_{2}^{1-l_{1}} \chi_{4}^{1-l_{2}}}-A \\
& \quad+\frac{1}{4\left(\chi_{2}^{l_{1}}-\chi_{1}^{l_{1}}\right)^{\eta_{1}}\left(\chi_{4}^{l_{2}}-\chi_{3}^{l_{2}}\right)^{\eta_{2}} \mathfrak{F}_{\rho_{1}, \eta_{1}+1}^{\sigma_{1}}\left[\omega_{1}\left(\chi_{2}^{l_{1}}-\chi_{1}^{l_{1}}\right)^{\rho_{1}}\right] \mathfrak{F}_{\rho_{2}, \eta_{2}+1}^{\sigma_{2}}\left[\omega_{2}\left(\chi_{4}^{l_{2}}-\chi_{3}^{l_{2}}\right)^{\rho_{2}}\right]} \\
& \quad \times\left(\Im_{\rho, \eta,\left(\chi_{2}^{l_{1}}\right)^{-},\left(\chi_{3}^{l_{2}}\right)^{+}, \omega}\left(\frac{f_{g}\left(\chi_{1}^{l_{1}}, \chi_{4}^{l_{2}}\right)}{\chi_{1}^{1-l_{1}} \chi_{4}^{1-l_{2}}}\right)+\Im_{\rho, \eta,\left(\chi_{2}^{l_{1}}\right)^{-},\left(\chi_{4}^{l_{2}}\right)^{-,}, \omega}\left(\frac{f_{g}\left(\chi_{1}^{l_{1}}, \chi_{3}^{l_{2}}\right)}{\chi_{1}^{1-l_{1}} \chi_{3}^{1-l_{2}}}\right)\right.
\end{aligned}
$$




$$
\begin{aligned}
& \left.+\Im_{\rho, \eta,\left(\chi_{1}^{l_{1}}\right)^{+},\left(\chi_{4}^{l_{2}}\right)^{-}, \omega}^{\sigma}\left(\frac{f_{g}\left(\chi_{2}^{l_{1}}, \chi_{3}^{l_{2}}\right)}{\chi_{2}^{1-l_{1}} \chi_{3}^{1-l_{2}}}\right)+\Im_{\rho, \eta,\left(\chi_{1}^{l_{1}}\right)^{+},\left(\chi_{3}^{l_{2}}\right)^{+}, \omega}^{\sigma}\left(\frac{f_{g}\left(\chi_{2}^{l_{1}}, \chi_{4}^{l_{2}}\right)}{\chi_{2}^{1-l_{1}} \chi_{4}^{1-l_{2}}}\right)\right) \\
& \leq \frac{\left(\chi_{2}^{l_{1}}-\chi_{1}^{l_{1}}\right)\left(\chi_{4}^{l_{2}}-\chi_{3}^{l_{2}}\right)}{4 l_{1} l_{2} \mathfrak{F}_{\rho_{1}, \eta_{1}+1}^{\sigma_{1}}\left[\omega_{1}\left(\chi_{2}^{l_{1}}-\chi_{1}^{l_{1}}\right)^{\rho_{1}}\right] \mathfrak{F}_{\rho_{2}, \eta_{2}+1}^{\sigma_{2}}\left[\omega_{2}\left(\chi_{4}^{l_{2}}-\chi_{3}^{l_{2}}\right)^{\rho_{2}}\right]} \\
& \times\left(\int_{0}^{1} \int_{0}^{1}\left[\mathcal{B}\left(\xi_{1}, \xi_{2}\right)\right]^{p} d \xi_{1} d \xi_{2}\right)^{\frac{1}{p}}\left[\int \int _ { 0 } ^ { 1 } \int _ { 0 } ^ { 1 } \left(h_{1}\left(\xi_{1}\right) h_{2}\left(\xi_{2}\right)\left|\frac{\partial^{2} f}{\partial \xi_{1} \partial \xi_{2}}\left(\chi_{1}, \chi_{3}\right)\right|^{q}\right.\right. \\
& +h_{1}\left(\xi_{1}\right) h_{2}\left(1-\xi_{2}\right)\left|\frac{\partial^{2} f}{\partial \xi_{1} \partial \xi_{2}}\left(\chi_{1}, \chi_{4}\right)\right|^{q}+h_{1}\left(1-\xi_{1}\right) h_{2}\left(\xi_{2}\right)\left|\frac{\partial^{2} f}{\partial \xi_{1} \partial \xi_{2}}\left(\chi_{2}, \chi_{3}\right)\right|^{q} \\
& \left.\left.+h_{1}\left(1-\xi_{1}\right) h_{2}\left(1-\xi_{2}\right)\left|\frac{\partial^{2} f}{\partial \xi_{1} \partial \xi_{2}}\left(\chi_{2}, \chi_{4}\right)\right|^{q}\right) d \xi_{1} d \xi_{2}\right\}^{\frac{1}{q}} \\
& +\left\{\int _ { 0 } ^ { 1 } \int _ { 0 } ^ { 1 } \left(h_{1}\left(\xi_{1}\right) h_{2}\left(1-\xi_{2}\right)\left|\frac{\partial^{2} f}{\partial \xi_{1} \partial \xi_{2}}\left(\chi_{1}, \chi_{3}\right)\right|^{q}+h_{1}\left(\xi_{1}\right) h_{2}\left(\xi_{2}\right)\left|\frac{\partial^{2} f}{\partial \xi_{1} \partial \xi_{2}}\left(\chi_{1}, \chi_{4}\right)\right|^{q}\right.\right. \\
& +h_{1}\left(1-\xi_{1}\right) h_{2}\left(1-\xi_{2}\right)\left|\frac{\partial^{2} f}{\partial \xi_{1} \partial \xi_{2}}\left(\chi_{2}, \chi_{3}\right)\right|^{q} \\
& \left.\left.+h_{1}\left(1-\xi_{1}\right) h_{2}\left(\xi_{2}\right)\left|\frac{\partial^{2} f}{\partial \xi_{1} \partial \xi_{2}}\left(\chi_{2}, \chi_{4}\right)\right|^{q}\right) d \xi_{1} d \xi_{2}\right\}^{\frac{1}{q}} \\
& +\left\{\int _ { 0 } ^ { 1 } \int _ { 0 } ^ { 1 } \left(h_{1}\left(1-\xi_{1}\right) h_{2}\left(\xi_{2}\right)\left|\frac{\partial^{2} f}{\partial \xi_{1} \partial \xi_{2}}\left(\chi_{1}, \chi_{3}\right)\right|^{q}\right.\right. \\
& +h_{1}\left(1-\xi_{1}\right) h_{2}\left(1-\xi_{2}\right)\left|\frac{\partial^{2} f}{\partial \xi_{1} \partial \xi_{2}}\left(\chi_{1}, \chi_{4}\right)\right|^{q}+h_{1}\left(\xi_{1}\right) h_{2}\left(\xi_{2}\right)\left|\frac{\partial^{2} f}{\partial \xi_{1} \partial \xi_{2}}\left(\chi_{2}, \chi_{3}\right)\right|^{q} \\
& \left.\left.+h_{1}\left(\xi_{1}\right) h_{2}\left(1-\xi_{2}\right)\left|\frac{\partial^{2} f}{\partial \xi_{1} \partial \xi_{2}}\left(\chi_{2}, \chi_{4}\right)\right|^{q}\right) d \xi_{1} d \xi_{2}\right\}^{\frac{1}{q}} \\
& +\left\{\int _ { 0 } ^ { 1 } \int _ { 0 } ^ { 1 } \left(h_{1}\left(1-\xi_{1}\right) h_{2}\left(1-\xi_{2}\right)\left|\frac{\partial^{2} f}{\partial \xi_{1} \partial \xi_{2}}\left(\chi_{1}, \chi_{3}\right)\right|^{q}\right.\right. \\
& +h_{1}\left(1-\xi_{1}\right) h_{2}\left(\xi_{2}\right)\left|\frac{\partial^{2} f}{\partial \xi_{1} \partial \xi_{2}}\left(\chi_{1}, \chi_{4}\right)\right|^{q}+h_{1}\left(\xi_{1}\right) h_{2}\left(1-\xi_{2}\right)\left|\frac{\partial^{2} f}{\partial \xi_{1} \partial \xi_{2}}\left(\chi_{2}, \chi_{3}\right)\right|^{q} \\
& \left.\left.\left.+h_{1}\left(\xi_{1}\right) h_{2}\left(\xi_{2}\right)\left|\frac{\partial^{2} f}{\partial \xi_{1} \partial \xi_{2}}\left(\chi_{2}, \chi_{4}\right)\right|^{q}\right) d \xi_{1} d \xi_{2}\right\}^{\frac{1}{q}}\right]
\end{aligned}
$$

where $\mathcal{B}\left(\xi_{1}, \xi_{2}\right)$ and $A$ are defined as in (3.16) and (3.17), respectively, and $f_{g}(x, y)=$ $f\left(g_{1}(x), g_{2}(y)\right)$ with $g_{1}(x)=x^{\frac{1}{l_{1}}}$ and $g_{2}(y)=y^{\frac{1}{l_{2}}}$.

Proof Making use of Lemma 3.1 and the properties of modulus, we get

$$
\begin{aligned}
& \mid \frac{f\left(\chi_{1}, \chi_{3}\right)}{4 \chi_{1}^{1-l_{1}} \chi_{3}^{1-l_{2}}}+\frac{f\left(\chi_{1}, \chi_{4}\right)}{4 \chi_{1}^{1-l_{1}} \chi_{4}^{1-l_{2}}}+\frac{f\left(\chi_{2}, \chi_{3}\right)}{4 \chi_{2}^{1-l_{1}} \chi_{3}^{1-l_{2}}}+\frac{f\left(\chi_{2}, \chi_{4}\right)}{4 \chi_{2}^{1-l_{1}} \chi_{4}^{1-l_{2}}}-A \\
& \quad+\frac{1}{4\left(\chi_{2}^{l_{1}}-\chi_{1}^{l_{1}}\right)^{\eta_{1}}\left(\chi_{4}^{l_{2}}-\chi_{3}^{l_{2}}\right)^{\eta_{2}} \mathfrak{F}_{\rho_{1}, \eta_{1}+1}^{\sigma_{1}}\left[\omega_{1}\left(\chi_{2}^{l_{1}}-\chi_{1}^{l_{1}}\right)^{\rho_{1}}\right] \mathfrak{F}_{\rho_{2}, \eta_{2}+1}^{\sigma_{2}}\left[\omega_{2}\left(\chi_{4}^{l_{2}}-\chi_{3}^{l_{2}}\right)^{\rho_{2}}\right]} \\
& \quad \times\left(\Im_{\rho, \eta,\left(\chi_{2}^{l_{1}}\right)^{-},\left(\chi_{3}^{l_{2}}\right)^{+}, \omega}^{\sigma}\left(\frac{f_{g}\left(\chi_{1}^{l_{1}}, \chi_{4}^{l_{2}}\right)}{\chi_{1}^{1-l_{1}} \chi_{4}^{1-l_{2}}}\right)+\Im_{\rho, \eta,\left(\chi_{2}^{l_{1}}\right)^{-},\left(\chi_{4}^{l_{2}}\right)^{-}, \omega}\left(\frac{f_{g}\left(\chi_{1}^{l_{1}}, \chi_{3}^{l_{2}}\right)}{\chi_{1}^{1-l_{1}} \chi_{3}^{1-l_{2}}}\right)\right.
\end{aligned}
$$




$$
\begin{aligned}
& \left.+\Im_{\rho, \eta,\left(\chi_{1}^{l_{1}}\right)^{+},\left(\chi_{4}^{l_{2}}\right)^{-}, \omega}^{\sigma}\left(\frac{f_{g}\left(\chi_{2}^{l_{1}}, \chi_{3}^{l_{2}}\right)}{\chi_{2}^{1-l_{1}} \chi_{3}^{1-l_{2}}}\right)+\Im_{\rho, \eta,\left(\chi_{1}^{l_{1}}\right)^{+},\left(\chi_{3}^{l_{2}}\right)^{+}, \omega}^{\sigma}\left(\frac{f_{g}\left(\chi_{2}^{l_{1}}, \chi_{4}^{l_{2}}\right)}{\chi_{2}^{1-l_{1}} \chi_{4}^{1-l_{2}}}\right)\right) \mid \\
& \leq \frac{\left(\chi_{2}^{l_{1}}-\chi_{1}^{l_{1}}\right)\left(\chi_{4}^{l_{2}}-\chi_{3}^{l_{2}}\right)}{4 l_{1} l_{2} \mathfrak{F}_{\rho_{1}, \eta_{1}+1}^{\sigma_{1}}\left[\omega_{1}\left(\chi_{2}^{l_{1}}-\chi_{1}^{l_{1}}\right)^{\rho_{1}}\right] \mathfrak{F}_{\rho_{2}, \eta_{2}+1}^{\sigma_{2}}\left[\omega_{2}\left(\chi_{4}^{l_{2}}-\chi_{3}^{l_{2}}\right)^{\rho_{2}}\right]} \\
& \times\left(\int_{0}^{1} \int_{0}^{1} \mathcal{B}\left(\xi_{1}, \xi_{2}\right) \mid \frac{\partial^{2} f}{\partial \xi_{1} \partial \xi_{2}}\left(\left(\xi_{1} \chi_{1}^{l_{1}}+\left(1-\xi_{1}\right) \chi_{2}^{l_{1}}\right)^{\frac{1}{l_{1}}},\right.\right. \\
& \left.\left(\xi_{2} \chi_{3}^{l_{2}}+\left(1-\xi_{2}\right) \chi_{4}^{l_{2}}\right)^{\frac{1}{l_{2}}}\right) \mid d \xi_{1} d \xi_{2} \\
& +\int_{0}^{1} \int_{0}^{1} \mathcal{B}\left(\xi_{1}, \xi_{2}\right) \mid \frac{\partial^{2} f}{\partial \xi_{1} \partial \xi_{2}}\left(\left(\xi_{1} \chi_{1}^{l_{1}}+\left(1-\xi_{1}\right) \chi_{2}^{l_{1}}\right)^{\frac{1}{l_{1}}}\right. \\
& \left.\left(\left(1-\xi_{2}\right) \chi_{3}^{l_{2}}+\xi_{2} \chi_{4}^{l_{2}}\right)^{\frac{1}{l_{2}}}\right) \mid d \xi_{1} d \xi_{2} \\
& +\int_{0}^{1} \int_{0}^{1} \mathcal{B}\left(\xi_{1}, \xi_{2}\right) \mid \frac{\partial^{2} f}{\partial \xi_{1} \partial \xi_{2}}\left(\left(\left(1-\xi_{1}\right) \chi_{1}^{l_{1}}+\xi_{1} \chi_{2}^{l_{1}}\right)^{\frac{1}{l_{1}}}\right. \\
& \left.\left(\xi_{2} \chi_{3}^{l_{2}}+\left(1-\xi_{2}\right) \chi_{4}^{l_{2}}\right)^{\frac{1}{l_{2}}}\right) \mid d \xi_{1} d \xi_{2} \\
& +\int_{0}^{1} \int_{0}^{1} \mathcal{B}\left(\xi_{1}, \xi_{2}\right) \mid \frac{\partial^{2} f}{\partial \xi_{1} \partial \xi_{2}}\left(\left(\left(1-\xi_{1}\right) \chi_{1}^{l_{1}}+\xi_{1} \chi_{2}^{l_{1}}\right)^{\frac{1}{l_{1}}}\right. \\
& \left.\left.\left(\left(1-\xi_{2}\right) \chi_{3}^{l_{2}}+\xi_{2} \chi_{4}^{l_{2}}\right)^{\frac{1}{l_{2}}}\right) \mid d \xi_{1} d \xi_{2}\right) \text {. }
\end{aligned}
$$

Making use of the $\left(l_{1}, h_{1}\right)-\left(l_{2}, h_{2}\right)$-convexity of $\left|\frac{\partial^{2} f}{\partial \xi_{1} \partial \xi_{2}}\right|^{q}$ on coordinates and Hölder's inequality, we obtain

$$
\begin{aligned}
& \mid \frac{f\left(\chi_{1}, \chi_{3}\right)}{4 \chi_{1}^{1-l_{1}} \chi_{3}^{1-l_{2}}}+\frac{f\left(\chi_{1}, \chi_{4}\right)}{4 \chi_{1}^{1-l_{1}} \chi_{4}^{1-l_{2}}}+\frac{f\left(\chi_{2}, \chi_{3}\right)}{4 \chi_{2}^{1-l_{1}} \chi_{3}^{1-l_{2}}}+\frac{f\left(\chi_{2}, \chi_{4}\right)}{4 \chi_{2}^{1-l_{1}} \chi_{4}^{1-l_{2}}}-A \\
& +\frac{1}{4\left(\chi_{2}^{l_{1}}-\chi_{1}^{l_{1}}\right)^{\eta_{1}}\left(\chi_{4}^{l_{2}}-\chi_{3}^{l_{2}}\right)^{\eta_{2}} \mathfrak{F}_{\rho_{1}, \eta_{1}+1}^{\sigma_{1}}\left[\omega_{1}\left(\chi_{2}^{l_{1}}-\chi_{1}^{l_{1}}\right)^{\rho_{1}}\right] \mathfrak{F}_{\rho_{2}, \eta_{2}+1}^{\sigma_{2}}\left[\omega_{2}\left(\chi_{4}^{l_{2}}-\chi_{3}^{l_{2}}\right)^{\rho_{2}}\right]} \\
& \times\left(\Im_{\rho, \eta,\left(\chi_{2}^{l_{1}}\right)^{-},\left(\chi_{3}^{l_{2}}\right)^{+}, \omega}\left(\frac{f_{g}\left(\chi_{1}^{l_{1}}, \chi_{4}^{l_{2}}\right)}{\chi_{1}^{1-l_{1}} \chi_{4}^{1-l_{2}}}\right)+\Im_{\rho, \eta,\left(\chi_{2}^{l_{1}}\right)^{-},\left(\chi_{4}^{l_{2}}\right)^{-}, \omega}\left(\frac{f_{g}\left(\chi_{1}^{l_{1}}, \chi_{3}^{l_{2}}\right)}{\chi_{1}^{1-l_{1}} \chi_{3}^{1-l_{2}}}\right)\right. \\
& \left.+\Im_{\rho, \eta,\left(\chi_{1}^{l_{1}}\right)^{+},\left(\chi_{4}^{l_{2}}\right)^{-}, \omega}^{\sigma}\left(\frac{f_{g}\left(\chi_{2}^{l_{1}}, \chi_{3}^{l_{2}}\right)}{\chi_{2}^{1-l_{1}} \chi_{3}^{1-l_{2}}}\right)+\Im_{\rho, \eta,\left(\chi_{1}^{l_{1}}\right)^{+},\left(\chi_{3}^{l_{2}}\right)^{+}, \omega}^{\sigma}\left(\frac{f_{g}\left(\chi_{2}^{l_{1}}, \chi_{4}^{l_{2}}\right)}{\chi_{2}^{1-l_{1}} \chi_{4}^{1-l_{2}}}\right)\right) \mid \\
& \leq \frac{\left(\chi_{2}^{l_{1}}-\chi_{1}^{l_{1}}\right)\left(\chi_{4}^{l_{2}}-\chi_{3}^{l_{2}}\right)}{4 l_{1} l_{2} \mathfrak{F}_{\rho_{1}, \eta_{1}+1}^{\sigma_{1}}\left[\omega_{1}\left(\chi_{2}^{l_{1}}-\chi_{1}^{l_{1}}\right)^{\rho_{1}}\right] \mathfrak{F}_{\rho_{2}, \eta_{2}+1}^{\sigma_{2}}\left[\omega_{2}\left(\chi_{4}^{l_{2}}-\chi_{3}^{l_{2}}\right)^{\rho_{2}}\right]} \\
& \times\left(\int_{0}^{1} \int_{0}^{1}\left[\mathcal{B}\left(\xi_{1}, \xi_{2}\right)\right]^{p} d \xi_{1} d \xi_{2}\right)^{\frac{1}{p}} \\
& \times\left(\left\{\int_{0}^{1} \int_{0}^{1}\left|\frac{\partial^{2} f}{\partial \xi_{1} \partial \xi_{2}}\left(\left(\xi_{1} \chi_{1}^{l_{1}}+\left(1-\xi_{1}\right) \chi_{2}^{l_{1}}\right)^{\frac{1}{l_{1}}},\left(\xi_{2} \chi_{3}^{l_{2}}+\left(1-\xi_{2}\right) \chi_{4}^{l_{2}}\right)^{\frac{1}{l_{2}}}\right)\right|^{q} d \xi_{1} d \xi_{2}\right\}^{\frac{1}{q}}\right. \\
& +\left\{\int_{0}^{1} \int_{0}^{1}\left|\frac{\partial^{2} f}{\partial \xi_{1} \partial \xi_{2}}\left(\left(\xi_{1} \chi_{1}^{l_{1}}+\left(1-\xi_{1}\right) \chi_{2}^{l_{1}}\right)^{\frac{1}{l_{1}}},\left(\left(1-\xi_{2}\right) \chi_{3}^{l_{2}}+\xi_{2} \chi_{4}^{l_{2}}\right)^{\frac{1}{l_{2}}}\right)\right|^{q} d \xi_{1} d \xi_{2}\right\}^{\frac{1}{q}}
\end{aligned}
$$




$$
\begin{aligned}
& +\left\{\int_{0}^{1} \int_{0}^{1}\left|\frac{\partial^{2} f}{\partial \xi_{1} \partial \xi_{2}}\left(\left(\left(1-\xi_{1}\right) \chi_{1}^{l_{1}}+\xi_{1} \chi_{2}^{l_{1}}\right)^{\frac{1}{l_{1}}},\left(\xi_{2} \chi_{3}^{l_{2}}+\left(1-\xi_{2}\right) \chi_{4}^{l_{2}}\right)^{\frac{1}{l_{2}}}\right)\right|^{q} d \xi_{1} d \xi_{2}\right\}^{\frac{1}{q}} \\
& \left.+\left\{\int_{0}^{1} \int_{0}^{1}\left|\frac{\partial^{2} f}{\partial \xi_{1} \partial \xi_{2}}\left(\left(\left(1-\xi_{1}\right) \chi_{1}^{l_{1}}+\xi_{1} \chi_{2}^{l_{1}}\right)^{\frac{1}{l_{1}}},\left(\left(1-\xi_{2}\right) \chi_{3}^{l_{2}}+\xi_{2} \chi_{4}^{l_{2}}\right)^{\frac{1}{l_{2}}}\right)\right|^{q} d \xi_{1} d \xi_{2}\right\}^{\frac{1}{q}}\right) \\
& \leq \frac{\left(\chi_{2}^{l_{1}}-\chi_{1}^{l_{1}}\right)\left(\chi_{4}^{l_{2}}-\chi_{3}^{l_{2}}\right)}{4 l_{1} l_{2} \mathfrak{F}_{\rho_{1}, \eta_{1}+1}^{\sigma_{1}}\left[\omega_{1}\left(\chi_{2}^{l_{1}}-\chi_{1}^{l_{1}}\right)^{\rho_{1}}\right] \mathfrak{F}_{\rho_{2}, \eta_{2}+1}^{\sigma_{2}}\left[\omega_{2}\left(\chi_{4}^{l_{2}}-\chi_{3}^{l_{2}}\right)^{\rho_{2}}\right]} \\
& \times\left(\int_{0}^{1} \int_{0}^{1}\left[\mathcal{B}\left(\xi_{1}, \xi_{2}\right)\right]^{p} d \xi_{1} d \xi_{2}\right)^{\frac{1}{p}}\left[\left\{\int _ { 0 } ^ { 1 } \int _ { 0 } ^ { 1 } \left(h_{1}\left(\xi_{1}\right) h_{2}\left(\xi_{2}\right)\left|\frac{\partial^{2} f}{\partial \xi_{1} \partial \xi_{2}}\left(\chi_{1}, \chi_{3}\right)\right|^{q}\right.\right.\right. \\
& +h_{1}\left(\xi_{1}\right) h_{2}\left(1-\xi_{2}\right)\left|\frac{\partial^{2} f}{\partial \xi_{1} \partial \xi_{2}}\left(\chi_{1}, \chi_{4}\right)\right|^{q}+h_{1}\left(1-\xi_{1}\right) h_{2}\left(\xi_{2}\right)\left|\frac{\partial^{2} f}{\partial \xi_{1} \partial \xi_{2}}\left(\chi_{2}, \chi_{3}\right)\right|^{q} \\
& \left.\left.+h_{1}\left(1-\xi_{1}\right) h_{2}\left(1-\xi_{2}\right)\left|\frac{\partial^{2} f}{\partial \xi_{1} \partial \xi_{2}}\left(\chi_{2}, \chi_{4}\right)\right|^{q}\right) d \xi_{1} d \xi_{2}\right\}^{\frac{1}{q}} \\
& +\left\{\int _ { 0 } ^ { 1 } \int _ { 0 } ^ { 1 } \left(h_{1}\left(\xi_{1}\right) h_{2}\left(1-\xi_{2}\right)\left|\frac{\partial^{2} f}{\partial \xi_{1} \partial \xi_{2}}\left(\chi_{1}, \chi_{3}\right)\right|^{q}+h_{1}\left(\xi_{1}\right) h_{2}\left(\xi_{2}\right)\left|\frac{\partial^{2} f}{\partial \xi_{1} \partial \xi_{2}}\left(\chi_{1}, \chi_{4}\right)\right|^{q}\right.\right. \\
& +h_{1}\left(1-\xi_{1}\right) h_{2}\left(1-\xi_{2}\right)\left|\frac{\partial^{2} f}{\partial \xi_{1} \partial \xi_{2}}\left(\chi_{2}, \chi_{3}\right)\right|^{q} \\
& \left.\left.+h_{1}\left(1-\xi_{1}\right) h_{2}\left(\xi_{2}\right)\left|\frac{\partial^{2} f}{\partial \xi_{1} \partial \xi_{2}}\left(\chi_{2}, \chi_{4}\right)\right|^{q}\right) d \xi_{1} d \xi_{2}\right\}^{\frac{1}{q}} \\
& +\left\{\int _ { 0 } ^ { 1 } \int _ { 0 } ^ { 1 } \left(h_{1}\left(1-\xi_{1}\right) h_{2}\left(\xi_{2}\right)\left|\frac{\partial^{2} f}{\partial \xi_{1} \partial \xi_{2}}\left(\chi_{1}, \chi_{3}\right)\right|^{q}\right.\right. \\
& +h_{1}\left(1-\xi_{1}\right) h_{2}\left(1-\xi_{2}\right)\left|\frac{\partial^{2} f}{\partial \xi_{1} \partial \xi_{2}}\left(\chi_{1}, \chi_{4}\right)\right|^{q} \\
& \left.\left.+h_{1}\left(\xi_{1}\right) h_{2}\left(\xi_{2}\right)\left|\frac{\partial^{2} f}{\partial \xi_{1} \partial \xi_{2}}\left(\chi_{2}, \chi_{3}\right)\right|^{q}+h_{1}\left(\xi_{1}\right) h_{2}\left(1-\xi_{2}\right)\left|\frac{\partial^{2} f}{\partial \xi_{1} \partial \xi_{2}}\left(\chi_{2}, \chi_{4}\right)\right|^{q}\right) d \xi_{1} d \xi_{2}\right\}^{\frac{1}{q}} \\
& +\left\{\int _ { 0 } ^ { 1 } \int _ { 0 } ^ { 1 } \left(h_{1}\left(1-\xi_{1}\right) h_{2}\left(1-\xi_{2}\right)\left|\frac{\partial^{2} f}{\partial \xi_{1} \partial \xi_{2}}\left(\chi_{1}, \chi_{3}\right)\right|^{q}\right.\right. \\
& +h_{1}\left(1-\xi_{1}\right) h_{2}\left(\xi_{2}\right)\left|\frac{\partial^{2} f}{\partial \xi_{1} \partial \xi_{2}}\left(\chi_{1}, \chi_{4}\right)\right|^{q}+h_{1}\left(\xi_{1}\right) h_{2}\left(1-\xi_{2}\right)\left|\frac{\partial^{2} f}{\partial \xi_{1} \partial \xi_{2}}\left(\chi_{2}, \chi_{3}\right)\right|^{q} \\
& \left.\left.\left.+h_{1}\left(\xi_{1}\right) h_{2}\left(\xi_{2}\right)\left|\frac{\partial^{2} f}{\partial \xi_{1} \partial \xi_{2}}\left(\chi_{2}, \chi_{4}\right)\right|^{q}\right) d \xi_{1} d \xi_{2}\right\}^{\frac{1}{q}}\right] \text {. }
\end{aligned}
$$

This rearranges to the proof of Theorem 3.4

Corollary 3.2 Theorem 3.4 with $\left|\frac{\partial^{2} f}{\partial \xi_{1} \partial \xi_{2}}\right|^{q} \leq K$, give the new inequality:

$$
\begin{aligned}
& \mid \frac{f\left(\chi_{1}, \chi_{3}\right)}{4 \chi_{1}^{1-l_{1}} \chi_{3}^{1-l_{2}}}+\frac{f\left(\chi_{1}, \chi_{4}\right)}{4 \chi_{1}^{1-l_{1}} \chi_{4}^{1-l_{2}}}+\frac{f\left(\chi_{2}, \chi_{3}\right)}{4 \chi_{2}^{1-l_{1}} \chi_{3}^{1-l_{2}}}+\frac{f\left(\chi_{2}, \chi_{4}\right)}{4 \chi_{2}^{1-l_{1}} \chi_{4}^{1-l_{2}}}-A \\
& \quad+\frac{1}{4\left(\chi_{2}^{l_{1}}-\chi_{1}^{l_{1}}\right)^{\eta_{1}}\left(\chi_{4}^{l_{2}}-\chi_{3}^{l_{2}}\right)^{\eta_{2}} \mathfrak{F}_{\rho_{1}, \eta_{1}+1}^{\sigma_{1}}\left[\omega_{1}\left(\chi_{2}^{l_{1}}-\chi_{1}^{l_{1}}\right)^{\rho_{1}}\right] \mathfrak{F}_{\rho_{2}, \eta_{2}+1}^{\sigma_{2}}\left[\omega_{2}\left(\chi_{4}^{l_{2}}-\chi_{3}^{l_{2}}\right)^{\rho_{2}}\right]} \\
& \quad \times\left(\Im_{\rho, \eta,\left(\chi_{2}^{l_{1}}\right)^{-},\left(\chi_{3}^{l_{2}}\right)^{+}, \omega}\left(\frac{f_{g}\left(\chi_{1}^{l_{1}}, \chi_{4}^{l_{2}}\right)}{\chi_{1}^{1-l_{1}} \chi_{4}^{1-l_{2}}}\right)+\Im_{\rho, \eta,\left(\chi_{2}^{l_{1}}\right)^{-},\left(\chi_{4}^{l_{2}}\right)^{-}, \omega}\left(\frac{f_{g}\left(\chi_{1}^{l_{1}}, \chi_{3}^{l_{2}}\right)}{\chi_{1}^{1-l_{1}} \chi_{3}^{1-l_{2}}}\right)\right.
\end{aligned}
$$




$$
\begin{aligned}
& \left.+\Im_{\rho, \eta,\left(\chi_{1}^{l_{1}}\right)^{+},\left(\chi_{4}^{l_{2}}\right)^{-,}, \omega}^{\sigma}\left(\frac{f_{g}\left(\chi_{2}^{l_{1}}, \chi_{3}^{l_{2}}\right)}{\chi_{2}^{1-l_{1}} \chi_{3}^{1-l_{2}}}\right)+\Im_{\rho, \eta,\left(\chi_{1}^{l_{1}}\right)^{+},\left(\chi_{3}^{l_{2}}\right)^{+}, \omega}^{\sigma}\left(\frac{f_{g}\left(\chi_{2}^{l_{1}}, \chi_{4}^{l_{2}}\right)}{\chi_{2}^{1-l_{1}} \chi_{4}^{1-l_{2}}}\right)\right) \mid \\
& \leq \frac{K\left(\chi_{2}^{l_{1}}-\chi_{1}^{l_{1}}\right)\left(\chi_{4}^{l_{2}}-\chi_{3}^{l_{2}}\right)}{l_{1} l_{2} \mathfrak{F}_{\rho_{1}, \eta_{1}+1}^{\sigma_{1}}\left[\omega_{1}\left(\chi_{2}^{l_{1}}-\chi_{1}^{l_{1}}\right)^{\rho_{1}}\right] \mathfrak{F}_{\rho_{2}, \eta_{2}+1}^{\sigma_{2}}\left[\omega_{2}\left(\chi_{4}^{l_{2}}-\chi_{3}^{l_{2}}\right)^{\rho_{2}}\right]} \\
& \times\left(\int_{0}^{1} \int_{0}^{1}\left[\mathcal{B}\left(\xi_{1}, \xi_{2}\right)\right]^{p} d \xi_{1} d \xi_{2}\right)^{\frac{1}{p}} \\
& \times\left(\int_{0}^{1} \int_{0}^{1}\left(h_{1}\left(1-\xi_{1}\right)+h_{1}\left(\xi_{1}\right)\right)\left(h_{2}\left(1-\xi_{2}\right)+h_{2}\left(\xi_{2}\right)\right) d \xi_{1} d \xi_{2}\right)^{\frac{1}{q}} \text {. }
\end{aligned}
$$

\section{Conclusion}

Since convexity has wide applications in many mathematical areas, the general class of $\left(l_{1}, h_{1}\right)-\left(l_{2}, h_{2}\right)$-convex functions on coordinates can be applied to obtain several results in convex analysis, special functions, related optimization theory, mathematical inequalities and may stimulate further research in different areas of pure and applied sciences.

\section{Acknowledgements}

We express our special thanks to the editor and referees.

\section{Funding}

Not applicable.

\section{Availability of data and materials}

Not applicable.

\section{Competing interests}

The authors declare that they have no competing interests.

\section{Authors' contributions}

All authors contributed equally and significantly in writing this article. All authors read and approved the final manuscript.

\section{Author details}

'Institute of Space Sciences, P.O. Box, MG-23, Magurele-Bucharest, R 76900, Romania. ${ }^{2}$ Department of Mathematics, Çankaya University, Ankara, Turkey. ${ }^{3}$ Department of Medical Research, China Medical University Hospital, China Medical University, Taichung, Taiwan. ${ }^{4}$ Department of Mathematics, Faculty of Technical Science, University Ismail Qemali, Vlora, Albania. ${ }^{5}$ Department of Mathematics, College of Education, University of Sulaimani, Sulaimani, Kurdistan Region, Iraq. ${ }^{6}$ Laboratoire des télécommunications, Faculté des Sciences et de la Technologie, University of 8 May 1945 Guelma, P.O. Box 401, 24000 Guelma, Algeria.

\section{Publisher's Note}

Springer Nature remains neutral with regard to jurisdictional claims in published maps and institutional affiliations.

Received: 14 August 2020 Accepted: 14 January 2021 Published online: 28 January 2021

\section{References}

1. Kilbas, A.A., Srivastava, H.M., Trujillo, J.J.: Theory and Applications of Fractional Differential Equations. Elsevier, Amsterdam (2006)

2. Atangana, A.: Fractional Operators with Constant and Variable Order with Application to Geo-Hydrology. Academic Press, New York (2017)

3. Srivastava, H.M., Mohammed, P.O.: A correlation between solutions of uncertain fractional forward difference equations and their paths. Front. Phys. 8, 280 (2020). https://doi.org/10.3389/fphy.2020.00280

4. Atangana, A., Baleanu, D.: New fractional derivatives with nonlocal and non-singular kernel: theory and application to heat transfer model. Therm. Sci. 20(2), 763-769 (2016)

5. Abdeljawad, T., Baleanu, D.: Integration by parts and its applications of a new nonlocal fractional derivative with Mittag-Leffler nonsingular kernel. J. Nonlinear Sci. Appl. 10(3), 1098-1107 (2017)

6. Mohammed, P.O., Abdeljawad, T., Jarad, F., Chu, Y.-M.: Existence and uniqueness of uncertain fractional backward difference equations of Riemann-Liouville type. Math. Probl. Eng. 2020, Article ID 6598682 (2020)

7. Alqudah, M.A., Mohammed, P.O., Abdeljawad, T.: Solution of singular integral equations via Riemann-Liouville fractional integrals. Math. Probl. Eng. 2020, Article ID 1250970 (2020)

8. Agarwal, P., Choi, J., Paris, R.B.: Extended Riemann-Liouville fractional derivative operator and its applications. J. Nonlinear Sci. Appl. 8(5), 451-466 (2015) 
9. Agarwal, P., Nieto, J.J., Luo, M.J.: Extended Riemann-Liouville type fractional derivative operator with applications. Open Math. 15(1), 1667-1681 (2017)

10. Yasemin, B., Baleanu, D.: Ostrowski type inequalities involving psi-Hilfer fractional integrals. Mathematics 7, 770 (2019)

11. Yasemin, B., Baleanu, D.: New aspects of Opial-type integral inequalities. Adv. Differ. Equ. 2018, 452 (2018)

12. Liu, X., Zhang, L., Agarwal, P., Wang, G.: On some new integral inequalities of Gronwall-Bellman-Bihari type with delay for discontinuous functions and their applications. Indag. Math. 27(1), 1-10 (2016)

13. Mehrez, K., Agarwal, P.: New Hermite-Hadamard type integral inequalities for convex functions and their applications. J. Comput. Appl. Math. 350, 274-285 (2019)

14. Agarwal, P., Tariboon, J., Ntouyas, S.K.: Some generalized Riemann-Liouville $k$-fractional integral inequalities. J. Inequal. Appl. 2016, 122 (2016)

15. Denton, Z., Vatsala, A.S.: Fractional integral inequalities and applications. Comput. Math. Appl. 59(3), 1087-1094 (2010)

16. Anastassiou, G.A.: Opial type inequalities involving Riemann-Liouville fractional derivatives of two functions with applications. Math. Comput. Model. 48, 344-374 (2008)

17. Fernandez, A., Mohammed, P.: Hermite-Hadamard inequalities in fractional calculus defined using Mittag-Leffler kernels. Math. Methods Appl. Sci., 1-18 (2020). https://doi.org/10.1002/mma.6188

18. Mohammed, P.O.: New integral inequalities for preinvex functions via generalized beta function. J. Interdiscip. Math. 22(4), 539-549 (2019)

19. Mohammed, P.O., Brevik, I.: A new version of the Hermite-Hadamard inequality for Riemann-Liouville fractional integrals. Symmetry 12, 610 (2020). https://doi.org/10.3390/sym12040610

20. Mohammed, P.O., Abdeljawad, T., Kashuri, A.: Fractional Hermite-Hadamard-Fejér inequalities for a convex function with respect to an increasing function involving a positive weighted symmetric function. Symmetry 12, 1503 (2020)

21. Mohammed, P.O., Abdeljawad, T., Zeng, S., Kashuri, A.: Fractional Hermite-Hadamard integral inequalities for a new class of convex functions. Symmetry 12, 1485 (2020)

22. Mohammed, P.O., Sarikaya, M.Z., Baleanu, D.: On the generalized Hermite-Hadamard inequalities via the tempered fractional integrals. Symmetry 12, 595 (2020). https://doi.org/10.3390/sym12040595

23. Mohammed, P.O.: Hermite-Hadamard inequalities for Riemann-Liouville fractional integrals of a convex function with respect to a monotone function. Math. Methods Appl. Sci., 1-11 (2019). https://doi.org/10.1002/mma.5784

24. Mohammed, P.O., Abdeljawad, T:: Modification of certain fractional integral inequalities for convex functions. Adv. Differ. Equ. 2020, 69 (2020)

25. Han, J., Mohammed, P.O., Zeng, H.: Generalized fractional integral inequalities of Hermite-Hadamard-type for a convex function. Open Math. 18(1), 794-806 (2020)

26. Mohammed, P.O., Sarikaya, M.Z.: Hermite-Hadamard type inequalities for F-convex function involving fractional integrals. J. Inequal. Appl. 2018, 359 (2018)

27. Mohammed, P.O., Sarikaya, M.Z:: On generalized fractional integral inequalities for twice differentiable convex functions. J. Comput. Appl. Math. 372, 112740 (2020)

28. Abdeljawad, T., Ali, M.A., Mohammed, P.O., Kashuri, A.: On inequalities of Hermite-Hadamard-Mercer type involving Riemann-Liouville fractional integrals. AIMS Math. 5, 7316-7331 (2020)

29. Abdeljawad, T., Mohammed, P.O., Kashuri, A.: New modified conformable fractional integral inequalities of Hermite-Hadamard type with applications. J. Funct. Spaces 2020, Article ID 4352357 (2020)

30. Raina, R.K.: On generalized Wright's hypergeometric functions and fractional calculus operators. East Asian Math. J. 21(2), 191-203 (2005)

31. Tomar, M., Agarwal, P., Jleli, M., Samet, B.: Certain Ostrowski type inequalities for generalized s-convex functions. J. Nonlinear Sci. Appl. 10, 5947-5957 (2017)

32. Dragomir, S.S.: On the Hadamard's inequality for convex functions on the coordinates in a rectangle from the plane. Taiwan. J. Math. 5, 775-788 (2001)

33. Sarikaya, M.Z:: On the Hermite-Hadamard-type inequalities for co-ordinated convex function via fractional integrals. Integral Transforms Spec. Funct. 25(2), 134-147 (2014)

34. Noor, M.A., Noor, K.I., Iftikhar, S., Rashid, S., Awan, M.U.: Coordinated convex functions and inequalities. Appl. Math E-Notes 19, 189-198 (2019)

35. Latif, M.A., Alomari, M.: On Hadamard-type inequalities for $h$-convex functions on the co-ordinates. Int. J. Math. Anal. 3(33), 1645-1656 (2009)

36. Cao, H.: A new Hermite-Hadamard type inequality for coordinate convex function. J. Inequal. Appl. 2020, 162 (2020)

37. Raees, M., Anwar, M.: On Hermite-Hadamard type inequalities of coordinated $\left(p_{1}, h_{1}\right)-\left(p_{2}, h_{2}\right)$-convex functions via Katugampola fractional integrals. Filomat 33(15), 4785-4802 (2019)

38. Yaldız, H., Sarikaya, M.Z., Dahmani, Z.: On the Hermite-Hadamard-Féjer-type inequalities for co-ordinated convex functions via fractional integrals. Int. J. Optim. Control Theor. Appl. 7(2), 205-215 (2017)

39. Tunç, T., Sarikaya, M.Z.: On Hermite-Hadamard type inequalities via fractional integral operators. Filomat 33(3), 837-854 (2019)

40. Yang, W.: Hermite-Hadamard type inequalities for $\left(p_{1}, h_{1}\right)-\left(p_{2}, h_{2}\right)$-convex functions on the coordinates. Tamkang J. Math. 47(3), 289-322 (2016)

41. Akkurt, A., Sarıkaya, M.Z., Budak, H., Yıldırım, H.: On the Hadamard's type inequalities for co-ordinated convex functions via fractional integrals. J. King Saud Univ., Sci. 29, 380-387 (2017)

42. Mohammed, P.O.: Some new Hermite-Hadamard type inequalities for MT-convex functions on differentiable coordinates. J. King Saud Univ., Sci. 30, 258-262 (2018)

43. Kara, H., Budak, H., Kiris, M.E.: On Fejer type inequalities for co-ordinated hyperbolic $\rho$-convex functions. AIMS Math. 5(5), 4681-4701 (2020)

44. Noor, M.A., Awan, M.U., Noor, K.I.: Integral inequalities for two-dimensional pq-convex functions. Filomat 30(2), 343-351 (2016) 